\title{
SECOND-ORDER NECESSARY CONDITIONS FOR A STRONG LOCAL MINIMUM IN A CONTROL PROBLEM WITH GENERAL CONTROL CONSTRAINTS
}

\author{
HÉLÈNE FRANKOWSKA* AND NIKOLAI P. OSMOLOVSKII ${ }^{\dagger}$
}

\begin{abstract}
We establish some second-order necessary optimality conditions for strong local minima in the Mayer type optimal control problem with a general control constraint $U \subset \mathbb{R}^{m}$ and final state constraint described by a finite number of inequalities. In the difference with the main approaches of the existing literature, the second order tangents to $U$ and the second order linearization of the control system are used to derive the second-order necessary conditions. This framework allows to replace the control system at hand by a differential inclusion with convex right-hand side. Such a relaxation of control system, and the differential calculus of derivatives of set-valued maps lead to fairly general statements. We illustrate the results by considering the case of control constraints defined by inequalities involving functions with positively or linearly independent gradients of active constraints, but also in the cases where the known approaches do not apply.
\end{abstract}

Key words. Optimal control, end point constraints, general control constraints, second-order necessary conditions, second order tangents, differential inclusion.

AMS subject classifications. Primary 49K15; Secondary 34H05, 49K24.

1. Introduction. We investigate second-order necessary conditions for a strong local minimum of the Mayer optimal control problem:

$$
\text { Minimize } g_{0}(x(1))
$$

over trajectories of the control system

$$
\dot{x}(t)=f(x(t), u(t)), \quad x(0)=x_{0}, \quad u(t) \in U \quad \text { a.e. in }[0,1]
$$

satisfying the end-point constraints

$$
g_{i}(x(1)) \leq 0, \quad i=1, \ldots, k,
$$

where $U$ is a compact subset of $\mathbb{R}^{m}$.

The main novelty of the present work lies in dealing with the general control constraint $U$ in the presence of endpoint inequality constraints and without imposing any structural assumptions on optimal controls.

There is an important literature on the subject of second-order necessary conditions that was already discussed in our previous paper [6], and also in [7]-[10]. Let us underline that, usually, the second-order necessary conditions are derived by reducing the optimal control problem at hand to an infinite dimensional mathematical programming one. This technique, however, does not allow to work with general sets $U$ and often requests some structural properties of the optimal controls, such as, for instance, their piecewise continuity. At the same time, on one hand, no existence theorems guarantee that optimal controls have a particular structure and, under standard

*Corresponding author, CNRS, IMJ-PRG, UMR 7586, Sorbonne Universités, UPMC Univ Paris 06, case 247, 4 place Jussieu, 75252 Paris, France. tel. 33-(0)1-44-27-71-87, E-mail: helene.frankowska@imj-prg.fr.

${ }^{\dagger}$ University of Technology and Humanities, 26-600 Radom, ul. Malczewskiego 20A, Poland; Systems Research Institute, Polish Academy of Sciences, ul. Newelska 6, 01-447, Warszawa, Poland; Moscow State University of Civil Engineering, Jaroslavskoe shosse 26, Moscow, Russia . E-mail: osmolovski@uph.edu.pl. 
assumptions, they are merely Lebesgue measurable. On the other hand, the mathematical programming theory is appropriate only when $U$ is an intersection of sets defined by inequality and/or equality constraints.

These are the main reasons to develop an alternative approach that applies for measurable optimal controls and general sets $U$. Nevertheless, if a supplementary information about optimal controls is available, then some additional results can be derived. For instance, [11, Sections 6 and 7$]$ discuss jump conditions in the case when the control system is affine in the control variable, $U$ is a polytope and an optimal control is a piecewise constant mapping taking values at the vertices of $U$. Such investigations are out of scope of the present manuscript.

We use instead the second order variational analysis for arbitrary measurable optimal controls continuing the same straightforward approach as the one used in [6] for derivation of second-order necessary conditions for a weak local minimum in a problem without endpoint constraints. Even though, by an appropriate adaptation of the assumptions, the results of this paper can be extended to more general initial point constraints, non autonomous control systems and time dependent control constraints $u(t) \in U(t)$, we intentionally restricted our study to the autonomous case, a fixed initial condition and compact set of control constraints $U$, to keep the presentation less technical and the approach more limpid.

When final-point constraint is absent, then any measurable $u:[0,1] \rightarrow U$ (a control) is admissible and for this reason the variational approach may be applied to any perturbations of a given optimal control. In [6] we used this fact to derive second order conditions (associated to the maximum principle in the normal form) in a straightforward way. This is not, however the case when a final-point constraint is present. First of all the maximum principle may be abnormal and it may happen that for a control $u(\cdot)$ the corresponding trajectory $x(\cdot)$ of our control system satisfies $g_{i}(x(1))>0$ for some $i$. Hence some perturbations of controls are not allowed leading to difficulties in direct application of the variational method. To deal with this more general case and with strong local minima instead of the weak ones, in the present work we combine tools from [5] and the separation theorem.

Previously, in [7], the variational approach was applied to study weak local minima for a Bolza problem under a state constraint, but also in the absence of any finalpoint constraint. Adding such additional constraint to the problem considered in [7] is not a trivial extension. Consequently, in [5], for the Mayer problem with state and end-point constraints second order necessary conditions were derived for strong local minima satisfying the normal maximum principle. These necessary conditions are stated for second order variations of trajectories having final-points in a prescribed open set, see [5, Theorem 6.8]. That is the set of second order variations is narrowed down by an additional restriction. In the present work we do not assume that the maximum principle is normal and instead of second order variations of trajectories, our necessary conditions involve second order tangents to $U$, without any restrictions on these tangents. Let us recall that under some constraints qualification assumptions the maximum principle is normal.

In the main result of this paper, Theorem 3.2, we do not impose any constraints qualification assumptions and we state second order necessary conditions even when the maximum principle is abnormal.

Before formulating the main result, let us recall definitions of the first and second order tangents to a closed set $U \subset \mathbb{R}^{m}$ at a given point. 
The adjacent tangent cone to $U$ at a point $u_{0} \in U$ is the set

$$
T_{U}^{b}\left(u_{0}\right)=\left\{u \in \mathbb{R}^{m}: \lim _{h \rightarrow 0+} \frac{\operatorname{dist}_{U}\left(u_{0}+h u\right)}{h}=0\right\},
$$

where $\operatorname{dist}_{U}(u)=\min _{u^{\prime} \in U}\left|u^{\prime}-u\right|$. When $U$ is convex, to be in line with the usual notations of convex analysis, we will also write $T_{U}\left(u_{0}\right)$ instead of $T_{U}^{b}\left(u_{0}\right)$.

Now, let $u_{0} \in U$ and $u \in \mathbb{R}^{m}$. For every pair $\left(u_{0}, u\right)$, the second-order adjacent set to $U$ at $\left(u_{0}, u\right)$ is the set

$$
T_{U}^{b(2)}\left(u_{0}, u\right):=\left\{v \in \mathbb{R}^{m}: \lim _{h \rightarrow 0+} \frac{\operatorname{dist}_{U}\left(u_{0}+h u+h^{2} v\right)}{h^{2}}=0\right\} .
$$

Clearly, $v \in T_{U}^{b(2)}\left(u_{0}, u\right)$ if and only if for every $h>0$ there exists an element $r(h) \in$ $\mathbb{R}^{m}$ such that $u_{0}+h u+h^{2} v+r(h) \in U$ and $|r(h)|=o\left(h^{2}\right)$ (the latter automatically implies that $\left.u \in T_{U}^{b}\left(u_{0}\right)\right)$. Any such couple $(u, v)$ can be seen as a second order jet to $U$ at $u_{0}$ (in the sense that $u_{0}+h u+h^{2} v \in U+o\left(h^{2}\right) B$, where $B$ stands for the closed unit ball.)

Our approach is based on the following elementary facts: Let $\psi: \mathbb{R}^{m} \rightarrow \mathbb{R}$ be a $C^{2}$-function and let $u_{0} \in U$ be a point of a local minimum of $\psi$ on a set $U$. Then, obviously, the following inequality holds

$$
\psi^{\prime}\left(u_{0}\right) u \geq 0 \quad \forall u \in T_{U}^{\mathrm{b}}\left(u_{0}\right)
$$

(this is the first-order necessary condition for a local minimum of $\psi$ on a set $U$ at the point $u_{0}$ ), and, moreover, for any $u \in T_{U}^{b}\left(u_{0}\right)$ such that $\psi^{\prime}\left(u_{0}\right) u=0$ (such directions $u$ are called critical) the following is true:

$$
\psi^{\prime}\left(u_{0}\right) v+\frac{1}{2}\left\langle\psi^{\prime \prime}\left(u_{0}\right) u, u\right\rangle \geq 0 \quad \forall v \in T_{U}^{b(2)}\left(u_{0}, u\right)
$$

(this is the second-order necessary condition for a local minimum of $\psi$ on a set $U$ at the point $u_{0}$ ). All the difficulties are concentrated then on deducing from the above abstract inequality the second-order necessary optimality conditions in concrete situations by computing subsets of second order tangents. We would like to underline that, in general, we can compute only subsets of tangents and second order tangents to sets. However this is not restrictive for necessary optimality conditions: they say that for all vectors in first (resp. second) order tangents some inequalities are verified. Then it is also true for subsets of tangents. In particular, the Pontryagin maximum principle can be derived using a particular subset of tangents to the reachable set of control system.

The paper is organized as follows. In section 2 we state the control problem under consideration, recall the notion of a strong local minimum and the first-order necessary condition for a strong minimum - the Pontryagin minimum principle. Section 3 contains the main results of the paper - the second-order necessary conditions for a strong local minimum of the Mayer problem with constraint $u(t) \in U$. In Section 4 we recall all necessary results on differential inclusions and the first- and second-order adjacent derivatives of sets, used in Section 5 to prove the main theorems. In Section 6 we examine the case where the set $U$ is a convex polytope, and some other examples of $U$. In Section 7, we study the case, when the control set $U$ is given by a system of inequalities $\varphi_{i}(u) \leq 0, i=1, \ldots, q$, assuming here that all $\varphi_{i}$ are $C^{2}$ and the gradients of active constraints are positively independent. Finally, in Section 8, we refine the result, obtained in the previous section, for the case, when the gradients of active control constraints are linearly independent. 
2. Statement of the problem. Strong minimizers. First-order necessary conditions. Denote by $W^{1,1}\left([0,1], \mathbb{R}^{n}\right)$ the Sobolev space of absolutely continuous functions $x:[0,1] \rightarrow \mathbb{R}^{n}$ with the norm $\|x(\cdot)\|_{1,1}=|x(0)|+\int_{0}^{1}|\dot{x}(t)| \mathrm{d} t$ and by $L^{\infty}\left([0,1], \mathbb{R}^{m}\right)$ the space of measurable essentially bounded functions $u:[0,1] \rightarrow \mathbb{R}^{m}$ with the norm $\|u(\cdot)\|_{\infty}=\operatorname{ess} \sup _{[0,1]}|u(t)|$, where $|\cdot|$ denotes the Euclidean norm. Let

$$
\Xi:=W^{1,1}\left([0,1], \mathbb{R}^{n}\right) \times L^{\infty}\left([0,1], \mathbb{R}^{m}\right)
$$

be the product of these two spaces. Define the norm of an element $\xi(\cdot)=(x(\cdot), u(\cdot)) \in$ $\Xi$ as the sum of the norms $\|\xi(\cdot)\|=\|x(\cdot)\|_{1,1}+\|u(\cdot)\|_{\infty}$.

Consider the following optimal control problem in the space $\Xi$ :

$$
\begin{gathered}
\text { Minimize } J(x, u):=g_{0}(x(1)), \\
g_{i}(x(1)) \leq 0, \quad i=1, \ldots, k, \\
\dot{x}(t)=f(x(t), u(t)), \quad x(0)=x_{0}, \quad u(t) \in U \quad \text { a.e. in }[0,1] .
\end{gathered}
$$

Here all functions $g_{i}: \mathbb{R}^{n} \rightarrow \mathbb{R}, f: \mathbb{R}^{n} \times \mathbb{R}^{m} \rightarrow \mathbb{R}^{n}$ are assumed to be of class $C^{2}$, and $U \subset \mathbb{R}^{m}$ is a nonempty compact set. Any trajectory-control pair $(x, u) \in \Xi$ satisfying (2.2), (2.3) is called admissible

Let $(\bar{x}, \bar{u})$ be admissible. Recall that a weak local minimum is a local minimum in the space $\Xi$. Further, $(\bar{x}, \bar{u})$ is called a strong local minimizer if there exists an $\varepsilon>0$ such that $J(x, u) \geq J(\bar{x}, \bar{u})$ for any admissible $(x, u) \in \Xi$ such that $\|x-\bar{x}\|_{\infty}<\varepsilon$. Obviously, any strong local minimizer is a weak local minimizer.

Let us recall the well-known first-order necessary optimality condition for problem (2.1)-(2.3) at a point $(\bar{x}, \bar{u})$ : the Pontryagin minimum principle. To this end, we introduce the Hamiltonian

$$
\mathcal{H}(x, u, p)=p f(x, u),
$$

where $p \in \mathbb{R}^{n *}$ (by $\mathbb{R}^{n *}$ we denote the space of $n$-dimensional row-vectors), and the Lagrange function associated to the cost and end-point constraints

$$
l(x, \lambda)=\sum_{i=0}^{k} \lambda_{i} g_{i}(x),
$$

where $x \in \mathbb{R}^{n}, \lambda=\left(\lambda_{0}, \lambda_{1}, \ldots, \lambda_{k}\right) \in \mathbb{R}^{k+1}$. Denote by $W^{1, \infty}\left([0,1], \mathbb{R}^{n *}\right)$ the space of Lipschitz continuous functions $p:[0,1] \rightarrow \mathbb{R}^{n *}$, and let

$$
\Sigma^{k+1}=\left\{\lambda=\left(\lambda_{0}, \ldots, \lambda_{k}\right) \in \mathbb{R}_{+}^{k+1}: \sum_{i=0}^{k} \lambda_{i}=1\right\} .
$$

An admissible pair $(\bar{x}, \bar{u})$ satisfies the Pontryagin minimum principle if there exist $\lambda \in \Sigma^{k+1}$ and $p(\cdot) \in W^{1, \infty}\left([0,1], \mathbb{R}^{n *}\right)$ such that

$$
\lambda_{i} g_{i}(\bar{x}(1))=0, \quad i=1, \ldots, k,
$$




$$
\begin{gathered}
-\dot{p}(t)=\mathcal{H}_{x}(\bar{x}(t), \bar{u}(t), p(t)), \quad \text { for a.e. } \quad t \in[0,1], \\
\qquad(1)=l_{x}(\bar{x}(1), \lambda), \\
\min _{v \in U} \mathcal{H}(\bar{x}(t), v, p(t))=\mathcal{H}(\bar{x}(t), \bar{u}(t), p(t)), \quad \text { for a.e. } \quad t \in[0,1] .
\end{gathered}
$$

Note that each multiplier $\lambda$ uniquely defines the function $p(\cdot)$ as the solution of the adjoint equation (2.5) with the transversality condition (2.6).

As is well-known, the Pontryagin minimum principle is a necessary condition for a strong minimum, that is the following theorem holds.

THEOREM 2.1. If $(\bar{x}, \bar{u})$ is a strong local minimizer, then there exist $\lambda \in \Sigma^{k+1}$ and $p(\cdot) \in W^{1, \infty}\left([0,1], \mathbb{R}^{n *}\right)$ satisfying (2.4)-(2.7).

Denote by $M(\bar{x}, \bar{u})$ the set of all $(\lambda, p(\cdot))$ satisfying (2.4)-(2.7). If $(\lambda, p(\cdot)) \in$ $M(\bar{x}, \bar{u})$, then the minimum condition (2.7) implies

$$
\mathcal{H}_{u}[t] v \geq 0 \quad \forall v \in T_{U}^{b}(\bar{u}(t)) \text { for a.e. } t \in[0,1],
$$

where $[t]=(\bar{x}(t), \bar{u}(t), p(t))$. Denote by $\Lambda(\bar{x}, \bar{u})$ the set of all $(\lambda, p(\cdot))$ satisfying (2.4)(2.6) and (2.8). Clearly, $M(\bar{x}, \bar{u}) \subset \Lambda(\bar{x}, \bar{u})$. The condition $\Lambda(\bar{x}, \bar{u}) \neq \emptyset$ is called the "weak local minimum principle". It is the first-order necessary optimality condition for a weak local minimum in the problem (but we will not study weak local minimizers here).

3. Main result: second-order necessary optimality conditions. At a point $(\bar{x}, \bar{u}) \in \Xi$, define the critical cone $\mathcal{C}(\bar{x}, \bar{u})$ as a set of all pairs $\xi(\cdot)=(y(\cdot), u(\cdot)) \in$ $\Xi$ satisfying the following conditions

$$
\begin{aligned}
& g_{i}^{\prime}(\bar{x}(1)) y(1) \leq 0, \quad i \in I_{g} \cup\{0\}, \\
& \dot{y}(t)=f_{x}[t] y(t)+f_{u}[t] u(t) \quad \text { a.e. in }[0,1], \quad y(0)=0, \\
& u(t) \in T_{U}^{b}(\bar{u}(t)) \quad \text { a.e. in }[0,1],
\end{aligned}
$$

where $I_{g}=\left\{i \in\{1, \ldots, k\}: g_{i}(\bar{x}(1))=0\right\}$ is the set of all active indices and $[t]$ stands for $(\bar{x}(t), \bar{u}(t))$.

The elements of the critical cone $\mathcal{C}(\bar{x}, \bar{u})$ have the following important property.

Lemma 3.1. Let $\xi(\cdot)=(y(\cdot), u(\cdot)) \in \mathcal{C}(\bar{x}, \bar{u})$ and $(\lambda, p(\cdot)) \in \Lambda(\bar{x}, \bar{u})$. Then

$$
\lambda_{i} g_{i}^{\prime}(\bar{x}(1)) y(1)=0, \quad i=1, \ldots, k, \quad \mathcal{H}_{u}[t] u(t)=0 \quad \text { a.e. }
$$

Proof We have

$$
\frac{\mathrm{d}}{\mathrm{d} t}(p(t) y(t))=-p(t) f_{x}[t] y(t)+p(t)\left(f_{x}[t] y(t)+f_{u}[t] u(t)\right)=p(t) f_{u}[t] u(t) \quad \text { a.e. }
$$

implying that

$$
\int_{0}^{1} \mathcal{H}_{u}[t] u(t) \mathrm{d} t=p(1) y(1)-p(0) y(0)=l_{x}(\bar{x}(1), \lambda) y(1)=\sum_{i=0}^{k} \lambda_{i} g_{i}^{\prime}(\bar{x}(1)) y(1) .
$$


Consequently,

$$
\sum_{i=0}^{k} \lambda_{i} g_{i}^{\prime}(\bar{x}(1)) y(1)-\int_{0}^{1} \mathcal{H}_{u}[t] u(t) \mathrm{d} t=0 .
$$

Since, by (2.8), (3.1), all summands in the left-hand-side of this equality are nonpositive, it follows that all of them are equal to zero. Moreover, the conditions $\int_{0}^{1} \mathcal{H}_{u}[t] u(t) \mathrm{d} t=0$ and $\mathcal{H}_{u}[t] u(t) \geq 0$ a.e. also imply that $\mathcal{H}_{u}[t] u(t)=0$ a.e.

Hence, if, for some index $i \in\{0, \ldots, k\}$, there exists $(\lambda, p(\cdot)) \in \Lambda(\bar{x}, \bar{u})$ such that $\lambda_{i}>0$, then the inequality $g_{i}^{\prime}(\bar{x}(1)) y(1) \leq 0$ in the definition of the critical cone can be replaced by the equality $g_{i}^{\prime}(\bar{x}(1)) y(1)=0$ without affecting $\mathcal{C}(\bar{x}, \bar{u})$. Similarly for any $(\lambda, p(\cdot)) \in \Lambda(\bar{x}, \bar{u})$ the condition $\mathcal{H}_{u}[t] u(t)=0$ can be added to the definition of $\mathcal{C}(\bar{x}, \bar{u})$ without affecting $\mathcal{C}(\bar{x}, \bar{u})$.

Denote by $\mathcal{C}_{0}(\bar{x}, \bar{u})$ the set of all pairs $\xi(\cdot)=(y(\cdot), u(\cdot)) \in \mathcal{C}(\bar{x}, \bar{u})$ satisfying an additional requirement: there exist a real $h_{0}>0$ and a nonnegative integrable function $c(\cdot)$, depending on $u(\cdot)$, such that

$$
\operatorname{dist}_{U}(\bar{u}(t)+h u(t)) \leq c(t) h^{2} \quad \forall h \in\left[0, h_{0}\right], \quad \text { a.e. in }[0,1] .
$$

Note that the above condition automatically implies (3.3).

Remark 3.1. a) If for some $t \in[0,1]$ the set $T_{U}^{b(2)}(\bar{u}(t), u(t))$ is nonempty, then, by the very definition of this set, there exist $h_{t}>0$ and $c_{t} \geq 0$ such that

$$
\operatorname{dist}_{U}(\bar{u}(t)+h u(t)) \leq c_{t} h^{2} \quad \forall h \in\left[0, h_{t}\right] .
$$

However, in general, $h_{t}$ do not have a uniform positive bound from the below and $c_{t}$ may be not integrably bounded.

b) The fulfilment of (3.5) depends on: geometric properties of $U$, on the relation of the function $\bar{u}(\cdot)$ with the boundary $\partial U$ of $U$, and on the choice of $u(\cdot)$. If $\partial U$ is of class $C^{2}$ and for some $\varepsilon>0$ and a.e. $t$, either $\bar{u}(t) \in \partial U$ or dist $\partial U(\bar{u}(t))>\varepsilon$, then condition (3.5) holds true for any $(y(\cdot), u(\cdot)) \in \mathcal{C}(\bar{x}, \bar{u})$. It is also verified for any element of $\mathcal{C}(\bar{x}, \bar{u})$ whenever $U$ is a polytope and $\bar{u}$ takes values in the set of vertices of $U$. The list of such examples can be continued.

c) We would like to underline, that if the control system is affine in the control variable, that is

$$
f(x, u)=f_{0}(x)+G(x) u,
$$

where $f_{0}: \mathbb{R}^{n} \rightarrow \mathbb{R}^{n}$ and $G(\cdot)$ is an $n \times m$ matrix function on $\mathbb{R}^{n}$, then, by the minimum principle (2.7), we expect optimal controls to take values on $\partial U$.

For any $u(\cdot):[0,1] \rightarrow \mathbb{R}^{m}$ define

$$
\begin{aligned}
V^{2}(\bar{u}(\cdot), u(\cdot)):= & \left\{v(\cdot):[0,1] \rightarrow \mathbb{R}^{m}: v(\cdot)\right. \text { is measurable, } \\
& \left.f_{u}[\cdot] v(\cdot) \text { is integrable and } v(t) \in T_{U}^{b(2)}(\bar{u}(t), u(t)) \text { a.e. }\right\},
\end{aligned}
$$

and for any $(\lambda, p(\cdot)) \in M(\bar{x}, \bar{u})$ and $t \in[0,1]$,

$$
\Upsilon(u(t), p(t)):=\inf \left\{\mathcal{H}_{u}[t] v: v \in T_{U}^{b(2)}(\bar{u}(t), u(t))\right\} \in[-\infty,+\infty],
$$

where, by convention, $\inf _{\emptyset}=+\infty$. Observe that if $T_{U}^{b(2)}(\bar{u}(t), u(t))=\emptyset$ on a subset of $t \in[0,1]$ of positive measure, then $V^{2}(\bar{u}(\cdot), u(\cdot))=\emptyset$. 
Further, for any $(\lambda, p(\cdot)) \in M(\bar{x}, \bar{u})$ and $\xi(\cdot)=(y(\cdot), u(\cdot)) \in \Xi$, set

$$
\Omega(\xi, \lambda, p):=\frac{1}{2}\left\langle l_{x x}(\bar{x}(1), \lambda) y(1), y(1)\right\rangle+\frac{1}{2} \int_{0}^{1}\left\langle\mathcal{H}^{\prime \prime}[t] \xi(t), \xi(t)\right\rangle \mathrm{d} t,
$$

where $\mathcal{H}^{\prime \prime}$ is the Hessian of $\mathcal{H}$. Even though $p(\cdot)$ is uniquely defined by $\lambda$, since it is needed to define $\mathcal{H}^{\prime \prime}[t]$, we include it in the notation $\Omega(\xi, \lambda, p)$. Note that the functional $\Omega(\xi, \lambda, p)$ is quadratic in $\xi$.

Theorems 3.2 and 3.4 below are the main results of this paper.

THEOREM 3.2. If $(\bar{x}(\cdot), \bar{u}(\cdot))$ is a strong local minimizer, then, for any $\xi(\cdot)=$ $(y(\cdot), u(\cdot)) \in \mathcal{C}_{0}(\bar{x}, \bar{u})$ with $V^{2}(\bar{u}(\cdot), u(\cdot)) \neq \emptyset$, there exists $(\lambda, p(\cdot)) \in M(\bar{x}, \bar{u})$, for which the function $\Upsilon(u(\cdot), p(\cdot))$ is integrable and the following inequality holds true:

$$
\Omega(\xi, \lambda, p)+\int_{0}^{1} \Upsilon(u(t), p(t)) \mathrm{d} t \geq 0 .
$$

When $(\lambda, p(\cdot)) \in M(\bar{x}, \bar{u})$ is as in the above theorem with $\lambda_{0}=0$, the inequality (3.10) is abnormal, since $g_{0}$ is then absent in the definition of the Lagrangian $l$ and hence $\Omega, p(\cdot)$ do not depend on the cost function $g_{0}$, even though it is present in the definition of the critical cone. Observe that (2.4) and (3.4) imply immediately the following corollary.

Corollary 3.3. Let $(\bar{x}(\cdot), \bar{u}(\cdot))$ be a strong local minimizer and assume that there exists $\xi(\cdot)=(y(\cdot), u(\cdot)) \in \mathcal{C}_{0}(\bar{x}, \bar{u})$ satisfying $g_{i}^{\prime}(\bar{x}(1)) y(1)<0$ for all $i \in I_{g}$ and such that $V^{2}(\bar{u}(\cdot), u(\cdot)) \neq \emptyset$. Set $\lambda=(1,0, \ldots, 0)$ and let $p$ solve (2.5), (2.6). Then (2.7) is satisfied, $\Upsilon(u(\cdot), p(\cdot))$ is integrable and

$$
\frac{1}{2}\left\langle g_{0}^{\prime \prime}(\bar{x}(1)) y(1), y(1)\right\rangle+\frac{1}{2} \int_{0}^{1}\left\langle\mathcal{H}^{\prime \prime}[t] \xi(t), \xi(t)\right\rangle \mathrm{d} t+\int_{0}^{1} \Upsilon(u(t), p(t)) \mathrm{d} t \geq 0 .
$$

Similarly to the above, define

$$
\begin{aligned}
V_{c o}^{2}(\bar{u}(\cdot), u(\cdot)):= & \left\{v(\cdot):[0,1] \rightarrow \mathbb{R}^{m}: v(\cdot)\right. \text { is measurable, } \\
& \left.f_{u}[\cdot] v(\cdot) \text { is integrable and } v(t) \in T_{c o U}^{b(2)}(\bar{u}(t), u(t)) \text { a.e. }\right\}
\end{aligned}
$$

and

$$
\Upsilon_{c o}(u(t), p(t)):=\inf \left\{\mathcal{H}_{u}[t] v: v \in T_{c o U}^{b(2)}(\bar{u}(t), u(t))\right\} .
$$

TheOREm 3.4. Let $f$ be affine in the control variable, cf. (3.6), with $f_{0} \in C^{2}$, $G \in C^{2}$ and $(\bar{x}(\cdot), \bar{u}(\cdot))$ be a strong local minimizer. Consider any $\xi(\cdot)=(y(\cdot), u(\cdot)) \in$ $\Xi$ satisfying (3.1), (3.2) such that $V_{c o}^{2}(\bar{u}(\cdot), u(\cdot)) \neq \emptyset$ and for some $h_{0}>0$,

$$
\operatorname{dist}_{c o U}(\bar{u}(t)+h u(t)) \leq c(t) h^{2} \quad \forall h \in\left[0, h_{0}\right], \quad \text { a.e. in }[0,1],
$$

where $c:[0,1] \rightarrow \mathbb{R}_{+}$is an integrable function. Then there exists $(\lambda, p(\cdot)) \in M(\bar{x}, \bar{u})$ such that $\Upsilon_{c o}(u(\cdot), p(\cdot))$ is integrable and

$$
\Omega(\xi, \lambda, p)+\int_{0}^{1} \Upsilon_{c o}(u(t), p(t)) \mathrm{d} t \geq 0 .
$$


The next two sections are devoted to the proofs of Theorems 3.2 and 3.4 .

Remark 3.2. Let us discuss the interest of the above "convexified" conclusions. If the set $U^{\prime}$ of all isolated points of $U$ is nonempty and $\bar{u}(t) \in U^{\prime}$ a.e., then $T_{U}^{b}(\bar{u}(t))=$ $\{0\}$ a.e., and hence $\mathcal{C}_{0}(\bar{x}, \bar{u})=\mathcal{C}(\bar{x}, \bar{u})=\{0\}$. Moreover, $T_{U}^{b(2)}(\bar{u}(t), 0)=\{0\}$ a.e., and therefore $\Upsilon(0, p(t))=0$ a.e. In this case, condition (3.10) becomes trivial. More can be said when the control system (2.3) is affine in the control. Then, as it is stated in Theorem 3.4, everywhere in the formulation of the necessary conditions of Theorem 3.2, the set $U$ can be replaced by its convexification, and then the final result may be drastically improved because, on one hand, $T_{U}^{b}(\bar{u}(t)) \subset T_{c o U}^{b}(\bar{u}(t))$ a.e., and on the other hand any $(y(\cdot), u(\cdot)) \in \mathcal{C}_{0}(\bar{x}, \bar{u})$ satisfies condition (3.5) with $U$ replaced by coU. Consequently, conclusions of Theorem 3.4 hold true on a larger set of critical pairs than in Theorem 3.2. Note also that $T_{\text {coU }}^{b}(\bar{u}(t)) \neq\{0\}$ whenever coU is not a singleton.

Theorem 3.4 concerns the affine control system (3.6). It is deduced from Theorem 3.2 by exploiting the relation

$$
c o\left(f_{0}(x)+G(x) U\right)=f_{0}(x)+G(x) c o U .
$$

Theorem 3.2, in turn, uses a result from [5] whose proof is based on the FilippovWażewski relaxation theorem. In general, $\operatorname{co} f(x, U) \neq f(x, c o U)$ and for this reason Theorem 3.4 does not have its analogue for a general mapping $f$.

We discuss next how Theorem 3.4 can be applied to the Bolza problem

$$
\text { Minimize } \quad g_{0}(x(1))+\int_{0}^{1}(\ell(x(t))+L(x(t)) \Psi(u(t))) \mathrm{d} t
$$

over admissible trajectory-control pairs $(x, u) \in \Xi$ (that is satisfying (2.2) and (2.3)), where $\ell: \mathbb{R}^{n} \rightarrow \mathbb{R}, L: \mathbb{R}^{n} \rightarrow \mathbb{R}^{k}$ and $\Psi: \mathbb{R}^{m} \rightarrow \mathbb{R}^{k}$ are $C^{2}$-functions.

It is well known that the above problem can be rewritten as the Mayer's one:

$$
\text { Minimize } g_{0}(x(1))+z(1)
$$

over trajectory-control pairs $((z, x), u) \in W^{1,1}\left([0,1], \mathbb{R} \times \mathbb{R}^{n}\right) \times L^{\infty}\left([0,1], \mathbb{R}^{m}\right)$ satisfying $(2.2)$ and

$$
\left\{\begin{array}{lll}
\dot{z}(t)=\ell(x(t))+L(x(t))) \alpha(t), & z(0)=0 & \\
\dot{x}(t)=f_{0}(x(t))+G(x(t)) u(t), & x(0)=x_{0}, & (u(t), \alpha(t)) \in \tilde{U} \quad \text { a.e. in }[0,1],
\end{array}\right.
$$

where $\tilde{U}=\{(u, \Psi(u)) \mid u \in U\}$, that is $\tilde{U}$ is the graph of $\Psi_{\mid U}$ (restriction of $\Psi$ to $U)$. Observe that if a pair $(\bar{x}(\cdot), \bar{u}(\cdot))$ is a strong local minimizer for the above Bolza problem, then it is also a strong local minimizer for the associated Mayer problem to which Theorem 3.4 can be applied. Under convenient assumptions on $\Psi$, some second order tangents to $c o \tilde{U}$ can be found.

Finally, we would like to underline that if we can compute some closed subsets $C(t) \subset T_{c o U}^{b(2)}(\bar{u}(t), u(t))$ for a.e. $t \in[0,1]\left(\right.$ instead of $\left.T_{c o U}^{b(2)}(\bar{u}(t), u(t))\right)$ such that $C(\cdot)$ is measurable and has at least one integrable selection, then

$$
\Upsilon_{c o}(u(t), p(t)) \leq \inf \left\{\mathcal{H}_{u}[t] v: v \in C(t)\right\}=: \beta(t)
$$

Thus $\beta$ is integrable and from Theorem 3.4 the following inequality can be deduced

$$
\Omega(\xi, \lambda, p)+\int_{0}^{1} \beta(t) \mathrm{d} t \geq 0 .
$$

If this inequality does not hold, then $\bar{u}$ is not strongly locally optimal. 
4. Preliminaries. We start with some definitions and facts proved in [5]. Let $\left\{A_{h}\right\}_{h>0}$ be a family of sets in $\mathbb{R}^{n}$. Define the set $\operatorname{Liminf}_{h \rightarrow 0+} A_{h}$ as follows:

$$
v \in \operatorname{Liminf}_{h \rightarrow 0+} A_{h} \Leftrightarrow \lim _{h \rightarrow 0+} \operatorname{dist}_{A_{h}}(v)=0 .
$$

Then for $u \in U$ and $v \in \mathbb{R}^{m}$ we have

$$
T_{U}^{b}(u)=\operatorname{Liminf}_{h \rightarrow 0+} \frac{U-u}{h} \quad \text { and } \quad T_{U}^{b(2)}(u, v)=\operatorname{Liminf}_{h \rightarrow 0+} \frac{U-u-h v}{h^{2}} \quad \forall v \in \mathbb{R}^{m} .
$$

Recall that the adjacent derivative of a locally Lipschitz (in the Hausdorff metric) set-valued map $F: \mathbb{R}^{n} \rightsquigarrow \mathbb{R}^{n}$ at a point $(x, y) \in \operatorname{graph}(F)$, in the direction $u \in \mathbb{R}^{n}$, is defined by

$$
\mathrm{d} F(x, y)(u)=\operatorname{Liminf}_{h \rightarrow 0+} \frac{F(x+h u)-y}{h} .
$$

For $u_{1} \in \mathbb{R}^{n}$ and $v_{1} \in \mathrm{d} F(x, y)\left(u_{1}\right)$, the second-order adjacent derivative of the set valued map $F(\cdot)$ at $\left(x, y, u_{1}, v_{1}\right)$ in the direction $u_{2} \in \mathbb{R}^{n}$ is defined by

$$
\mathrm{d}^{2} F\left(x, y, u_{1}, v_{1}\right)\left(u_{2}\right)=\operatorname{Liminf}_{h \rightarrow 0+} \frac{F\left(x+h u_{1}+h^{2} u_{2}\right)-y-h v_{1}}{h^{2}} .
$$

In the other words,

$$
v_{2} \in \mathrm{d}^{2} F\left(x, y, u_{1}, v_{1}\right)\left(u_{2}\right) \Leftrightarrow \lim _{h \rightarrow 0+} \frac{1}{h^{2}} \operatorname{dist}_{F\left(x+h u_{1}+h^{2} u_{2}\right)}\left(y+h v_{1}+h^{2} v_{2}\right)=0 .
$$

Let us return to control system (2.3). For every $x \in \mathbb{R}^{n}$, let us set

$$
F(x):=\operatorname{co} f(x, U) .
$$

Then, $F(x)$ is a convex compact set for all $x \in \mathbb{R}^{n}$, and $F$ is locally Lipschitz continuous. Thus, for our mapping $F(\cdot)$, the following is true, cf. [3]: for any $(x, y) \in \operatorname{graph}(F)$ and $u \in \mathbb{R}^{m}$ the set $\mathrm{d} F(x, y)(u)$ is convex and

$$
\mathrm{d} F(x, y)(0)=T_{F(x)}(y), \quad \mathrm{d} F(x, y)(u)+T_{F(x)}(y)=\mathrm{d} F(x, y)(u) .
$$

Moreover, by [5, Proposition 2.11], for all $(x, y) \in \operatorname{graph}(F), v_{1} \in d F(x, y)\left(u_{1}\right)$ and for every $u_{2} \in \mathbb{R}^{n}$,

$$
\mathrm{d}^{2} F\left(x, y, u_{1}, v_{1}\right)\left(u_{2}\right)+T_{F(x)}(y)=\mathrm{d}^{2} F\left(x, y, u_{1}, v_{1}\right)\left(u_{2}\right),
$$

where, by convention, $A+\emptyset=\emptyset$ for any $A \subset \mathbb{R}^{n}$.

Fix a pair $(\bar{x}, \bar{u}) \in \Xi$ which solves (2.3). Then $\bar{x}$ satisfies the differential inclusion

$$
\dot{x}(t) \in F(x(t)), \quad \text { for a.e. } t \in[0,1], \quad x(0)=x_{0} .
$$

Further, define the set of admissible first order variations $\mathcal{V}^{(1)}(\bar{x})$ at $\bar{x}$ as follows: an absolutely continuous function $y(\cdot)$ belongs to $\mathcal{V}^{(1)}(\bar{x})$ if and only if

(i) $\dot{y}(t) \in \mathrm{d} F(\bar{x}(t), \dot{\bar{x}}(t))(y(t))$ for a.e. $t \in[0,1], y(0)=0$,

(ii) $\exists \alpha(\cdot) \in L^{1}\left([0,1], \mathbb{R}_{+}\right), \exists h_{0}>0$ such that for all $h \in\left[0, h_{0}\right]$ and a.e. $t \in[0,1]$,

$$
\operatorname{dist}_{F(\bar{x}(t)+h y(t))}(\dot{\bar{x}}(t)+h \dot{y}(t)) \leq \alpha(t) h^{2} .
$$


For a given $y \in \mathcal{V}^{(1)}(\bar{x})$, we abbreviate $(\bar{x}(t), \dot{\bar{x}}(t), y(t), \dot{y}(t))$ by $[t]$ and define the set of admissible second-order variations $\mathcal{V}^{(2)}(\bar{x}, y)$ as the set of all absolutely continuous functions $w:[0,1] \rightarrow \mathbb{R}^{n}$ satisfying

$$
\dot{w}(t) \in \mathrm{d}^{2} F[t](w(t)) \text { for a.e. } t \in[0,1], \quad w(0)=0 .
$$

Denote by $S$ the set of all $x \in W^{1,1}\left([0,1], \mathbb{R}^{n}\right)$ such that there exists $u \in L^{\infty}\left([0,1], \mathbb{R}^{m}\right)$ with $(x, u)$ satisfying $(2.3)$. Recall that, if $f$ is continuous, then, by a measurable selection theorem, see for instance [1, Theorem 8.2.10], the set $S$ coincides with the set of trajectories of the differential inclusion

$$
\dot{x}(t) \in f(x(t), U) \quad \text { a.e. in } \quad[0,1], \quad x(0)=x_{0} .
$$

Thus, by [5, Theorem 3.3], for every $w \in \mathcal{V}^{(2)}(\bar{x}, y)$ and for any sequence $h_{j} \rightarrow 0+$ there exists a sequence $x_{j} \in S, j=1, \ldots$, such that

$$
\frac{x_{j}-\bar{x}-h_{j} y}{h_{j}^{2}} \rightarrow w \quad \text { uniformly on }[0,1] \text { as } j \rightarrow \infty .
$$

It follows that

$$
x_{j}=\bar{x}+h_{j} y+h_{j}^{2} w+h_{j}^{2} r_{j}, \quad \text { where }\left\|r_{j}\right\|_{\infty} \rightarrow 0 \text { as } j \rightarrow \infty .
$$

This is a key auxiliary fact that will be used in proving our main results.

We would like to underline that in [5] $F$ is supposed to satisfy also a sublinear growth assumption. However, the same proof applies whenever $U$ is compact and $f \in C^{2}$ and so this last assumption may be skipped.

5. Proof of the main results. We first prove Theorem 3.2. So, let $(\bar{x}, \bar{u})$ be a strong local minimizer of the problem (2.1)-(2.3). Without loss of generality we may assume that

$$
g_{i}(\bar{x}(1))=0, \quad i=0,1, \ldots, k,
$$

that is all indices of endpoint inequality constrains are active and the cost is equal to zero at the reference point $(\bar{x}, \bar{u})$.

Suppose that the set $\mathcal{V}^{(1)}(\bar{x})$ is nonempty. A sufficient condition for it will be provided in Lemma 5.3 below. Consider any $y \in \mathcal{V}^{(1)}(\bar{x})$, satisfying

$$
g_{i}^{\prime}(\bar{x}(1)) y(1) \leq 0, \quad i=0, \ldots, k,
$$

any $w \in \mathcal{V}^{(2)}(\bar{x}, y)$, and sequences $\left\{x_{j}\right\},\left\{h_{j}\right\}$ and $\left\{r_{j}\right\}$ as in (4.3). Since $x_{j} \in S$ for every $j$, there is a sequence of controls $\left\{u_{j}\right\}$ such that $\left\{\left(x_{j}, u_{j}\right)\right\}$ solves system (2.3). Then, for any $i=0,1, \ldots, k$,

$$
\begin{aligned}
g_{i}\left(x_{j}(1)\right) & =g_{i}\left(\bar{x}(1)+h_{j} y(1)+h_{j}^{2} w(1)+h_{j}^{2} r_{j}(1)\right)=g_{i}(\bar{x}(1))+h_{j} g_{i}^{\prime}(\bar{x}(1)) y(1) \\
& +h_{j}^{2}\left(g_{i}^{\prime}(\bar{x}(1)) w(1)+\frac{1}{2}\left\langle g_{i}^{\prime \prime}(\bar{x}(1)) y(1), y(1)\right\rangle\right)+o\left(h_{j}^{2}\right) .
\end{aligned}
$$

Taking into account (5.1) and (5.2), we deduce that for all $i=0, \ldots, k$,

$$
g_{i}\left(x_{j}(1)\right) \leq h_{j}^{2}\left(g_{i}^{\prime}(\bar{x}(1)) w(1)+\frac{1}{2}\left\langle g_{i}^{\prime \prime}(\bar{x}(1)) y(1), y(1)\right\rangle\right)+o\left(h_{j}^{2}\right) .
$$


Now, we can prove the following important lemma.

Lemma 5.1. Let $(\bar{x}, \bar{u})$ be a strong local minimizer and $y \in \mathcal{V}^{(1)}(\bar{x})$ satisfies (5.2). Then the system of conditions

$$
g_{i}^{\prime}(\bar{x}(1)) w(1)+\frac{1}{2}\left\langle g_{i}^{\prime \prime}(\bar{x}(1)) y(1), y(1)\right\rangle<0, i=0, \ldots, k, \quad w \in \mathcal{V}^{(2)}(\bar{x}, y)
$$

is incompatible.

Proof Assume the contrary. Let $w$ satisfies (5.4). Consider the sequence $\left\{\left(x_{j}, u_{j}\right)\right\}$ which solves (2.3), where $\left\{x_{j}\right\}$ is as in (4.3) with the corresponding sequence $\left\{h_{j}\right\}$. According to (5.3) and (5.4), for this sequence we have $g_{i}\left(x_{j}(1)\right)<0$ for all $i=0, \ldots, k$ and all $j$ sufficiently large. This contradicts to the assumption that $(\bar{x}, \bar{u})$ is a strong local minimizer and ends the proof.

Fix $y \in \mathcal{V}^{(1)}(\bar{x})$ satisfying (5.2). Define the following open, possibly empty, affine half-spaces in $\mathbb{R}^{n}$ :

$$
Q_{i}=\left\{z \in \mathbb{R}^{n}: g_{i}^{\prime}(\bar{x}(1)) z+\frac{1}{2}\left\langle g_{i}^{\prime \prime}(\bar{x}(1)) y(1), y(1)\right\rangle<0\right\}, \quad i=0, \ldots, k .
$$

Further, set

$$
\mathcal{E}^{2}=\left\{w(1): \quad w(\cdot) \in \mathcal{V}^{(2)}(\bar{x}, y)\right\} .
$$

It follows from Lemma 5.1, that the condition

$$
\left(\bigcap_{i=0}^{k} Q_{i}\right) \bigcap \mathcal{E}^{2}=\emptyset
$$

is necessary for $(\bar{x}, \bar{u})$ to be a strong local minimizer. In general, the set $\mathcal{E}^{2}$ may be not convex. Let $\tilde{\mathcal{E}}^{2}$ be an arbitrary convex subset of $\mathcal{E}^{2}$. Then the condition

$$
\left(\bigcap_{i=0}^{k} Q_{i}\right) \bigcap \tilde{\mathcal{E}}^{2}=\emptyset
$$

is also necessary for $(\bar{x}, \bar{u})$ to be a strong local minimizer. Assume $\tilde{\mathcal{E}}^{2} \neq \emptyset$ and set

$$
\left(\tilde{\mathcal{E}}^{2}\right)^{\prime}=\left\{z^{*} \in \mathbb{R}^{n}: \inf \left\langle z^{*}, \tilde{\mathcal{E}}^{2}\right\rangle>-\infty .\right\},
$$

where $\left\langle z^{*}, \tilde{\mathcal{E}}^{2}\right\rangle$ is the image (in $\mathbb{R}$ ) of the set $\tilde{\mathcal{E}}^{2}$ by the linear functional $z^{*}$.

Suppose first that $Q_{i} \neq \emptyset$ for every $i$. According to the separation theorem, there exists a nontrivial pair $(\lambda, \pi)$ such that $\lambda=\left(\lambda_{0}, \ldots, \lambda_{k}\right) \in \mathbb{R}_{+}^{k+1}, \pi \in\left(\tilde{\mathcal{E}}^{2}\right)^{\prime}$ and

$$
\begin{gathered}
\pi=\sum_{i=0}^{k} \lambda_{i} g_{i}^{\prime}(\bar{x}(1)), \\
\frac{1}{2} \sum_{i=0}^{k} \lambda_{i}\left\langle g_{i}^{\prime \prime}(\bar{x}(1)) y(1), y(1)\right\rangle+\inf \left\langle\pi, \tilde{\mathcal{E}}^{2}\right\rangle \geq 0 .
\end{gathered}
$$

If $\lambda=0$, then it follows from (5.5) that $\pi=0$. Therefore, the nontriviality condition for the pair $(\lambda, \pi)$ is: $\sum_{i=0}^{k} \lambda_{i}>0$, which can be replaced by the normalized condition 
$\sum_{i=0}^{k} \lambda_{i}=1$. Then $\lambda \in \Sigma_{+}^{k+1}$. Using the Lagrange function $l$ introduced in Section 2, we can rewrite conditions (5.5) and (5.6) as follows: $\pi=l_{x}(\bar{x}(1), \lambda)$ and

$$
\frac{1}{2}\left\langle l_{x x}(\bar{x}(1), \lambda) y(1), y(1)\right\rangle+\inf \left\langle l_{x}(\bar{x}(1), \lambda), \tilde{\mathcal{E}}^{2}\right\rangle \geq 0 .
$$

If $Q_{i}=\emptyset$ for some $i$, then $g_{i}^{\prime}(\bar{x}(1))=0$ and $\left\langle g_{i}^{\prime}(\bar{x}(1)) y(1), y(1)\right\rangle \geq 0$. Setting in this case $\lambda_{i}=1$ and $\lambda_{j}=0$ for all $j \neq i$, we obtain: $\lambda \in \Sigma_{+}^{k+1}, l_{x}(\bar{x}(1), \lambda)=0$ and then (5.7) holds true again.

We have proved the following lemma.

Lemma 5.2. Let $y \in \mathcal{V}^{(1)}(\bar{x})$ satisfy (5.2). Then there exists $\lambda \in \Sigma_{+}^{k+1}$ such that inequality (5.7) holds true.

The question remains to find, in particular situations, convex sets $\tilde{\mathcal{E}}^{2}$, which are sufficiently "informative" to obtain the second-order necessary conditions and for which it is possible to calculate effectively the lower bound in (5.7).

Lemma 5.3. Let $y(\cdot)$ solve the "system in variations"

$$
\dot{y}(t)=f_{x}[t] y(t)+f_{u}[t] u(t), \quad y(0)=0,
$$

where $u(\cdot) \in L^{\infty}\left([0,1], \mathbb{R}^{m}\right)$ satisfies condition (3.5) with some integrable $c(\cdot) \geq 0$ and some $h_{0}>0$. Then $y(\cdot) \in \mathcal{V}^{(1)}(\bar{x})$.

Proof It follows directly from the definitions that for a.e. $t \in[0,1]$,

$$
f_{x}[t] y \in \mathrm{d} F(\bar{x}(t), f[t])(y) \text { and } f_{u}[t] u \in T_{F(\bar{x}(t))}(f[t]), \forall y \in \mathbb{R}^{n}, \forall u \in \mathbb{R}^{m} .
$$

This and the second relation in (4.1) imply that $\dot{y}(t) \in \mathrm{d} F(\bar{x}(t), \dot{\bar{x}}(t))(y(t))$ a.e., i.e., condition $(i)$ in the definition of the set $\mathcal{V}^{(1)}(\bar{x})$ is fulfilled. To show that condition (ii) is also fulfilled, we use assumption (3.5).

For every $h \in\left[0, h_{0}\right]$ and $t \in[0,1]$, let $u_{h}(t) \in U$ be such that $\mid \bar{u}(t)+h u(t)-$ $u_{h}(t) \mid \leq c(t) h^{2}$ a.e. and $u_{h}(\cdot)$ is measurable. The existence of such $u_{h}$ follows from the measurable selection theorem. Since $f\left(\bar{x}(t)+h y(t), u_{h}(t)\right) \in F(\bar{x}(t)+h y(t))$ for every $h \in\left[0, h_{0}\right]$, setting $r_{h}(t):=u_{h}(t)-(\bar{u}(t)+h u(t))$, we deduce that for a.e. $t \in[0,1]$,

$$
\begin{gathered}
\operatorname{dist}_{F(\bar{x}(t)+h y(t))}(\dot{\bar{x}}(t)+h \dot{y}(t)) \leq\left|f\left(\bar{x}(t)+h y(t), u_{h}(t)\right)-(\dot{\bar{x}}(t)+h \dot{y}(t))\right| \\
=\left|f\left(\bar{x}(t)+h y(t), \bar{u}(t)+h u(t)+r_{h}(t)\right)-\dot{\bar{x}}(t)-h \dot{y}(t)\right| .
\end{gathered}
$$

Since $u(\cdot)$ is essentially bounded and for a.e. $t \in[0,1]$ we have $\bar{u}(t)+h u(t)+r_{h}(t) \in$ $U$ for every $h \in\left[0, h_{0}\right]$, it follows that $\left\{\left\|r_{h}(\cdot)\right\|_{\infty}\right\}_{h \in\left[0, h_{0}\right]}$ is bounded. Using that $\left|r_{h}(t)\right| \leq c(t) h^{2}$ a.e. and that $f$ is locally Lipschitz, we deduce that for a constant $C \geq 0$, and for a.e. $t \in[0,1]$

$\left|f\left(\bar{x}(t)+h y(t), \bar{u}(t)+h u(t)+r_{h}(t)\right)-f(\bar{x}(t)+h y(t), \bar{u}(t)+h u(t))\right| \leq C c(t) h^{2} \quad \forall h \in\left[0, h_{0}\right]$.

Because $f \in C^{2}$ we conclude that there exists an integrable function $\alpha(\cdot) \geq 0$ such that for a.e. $t \in[0,1]$ and every $h \in\left[0, h_{0}\right]$,

$$
f\left(\bar{x}(t)+h y(t), \bar{u}(t)+h u(t)+r_{h}(t)\right)=f(\bar{x}(t), \bar{u}(t))+h f_{x}[t] y(t)+h f_{u}[t] u(t)+\gamma_{h}(t),
$$

where $\left|\gamma_{h}(t)\right| \leq \alpha(t) h^{2}$. Observe that the left hand side of this equality belongs to the set $F(\bar{x}(t)+h y(t))$ a.e., while the right hand side is equal to $\dot{\bar{x}}(t)+h \dot{y}(t)+\gamma_{h}(t)$ a.e. Hence $\operatorname{dist}_{F(\bar{x}(t)+h y(t))}(\dot{\bar{x}}(t)+h \dot{y}(t)) \leq \alpha(t) h^{2} \quad$ a.e. and therefore $y(\cdot) \in \mathcal{V}^{(1)}(\bar{x})$. 
Let $\xi(\cdot)=(y(\cdot), u(\cdot))$ be as in Lemma 5.3, and hence, $y \in \mathcal{V}^{(1)}(\bar{x})$. Consider the control system

$$
\dot{w}(t)=f_{x}[t] w(t)+f_{u}[t] v(t)+\frac{1}{2} f^{\prime \prime}[t] \xi(t) \xi(t)+\eta(t), \quad w(0)=0,
$$

where controls $v(\cdot), \eta(\cdot)$ satisfy

$$
v(\cdot) \in V^{2}(\bar{u}(\cdot), u(\cdot)), \quad \eta(\cdot) \text { is integrable, } \eta(t) \in T_{F(\bar{x}(t))}(\dot{\bar{x}}(t)) \text { a.e. }
$$

Define

$$
\tilde{\mathcal{E}}^{2}=\{w(1): w(\cdot) \text { solves (5.9) for some } v, \eta \text { as in (5.10) }\} .
$$

So, $\tilde{\mathcal{E}}^{2}$ depends on $\xi(\cdot)$. Recall that $\mathcal{E}^{2}$ depends on $y$, since this is so for $\mathcal{V}^{(2)}(\bar{x}, y)$.

Lemma 5.4. Let $\xi(\cdot)=(y(\cdot), u(\cdot))$ be as in Lemma 5.3 and the set $\tilde{\mathcal{E}}^{2}$ be as defined above. Then $\tilde{\mathcal{E}}^{2}$ is a convex subset of $\mathcal{E}^{2}$ (defined with the same $y$ ).

Proof Fix $\xi(\cdot)=(y(\cdot), u(\cdot))$ as in Lemma 5.3, and let $t \in[0,1]$ be such $\dot{y}(t)=$ $f_{x}[t] y(t)+f_{u}[t] u(t)$ and $\dot{\bar{x}}(t)=f[t]$. Observe, that the set of such $t$ has a full measure in $[0,1]$. Let $v \in T_{U}^{b(2)}(\bar{u}(t), u(t))$ and consider $r_{h}=o\left(h^{2}\right)$ such that $\bar{u}(t)+h u(t)+$ $h^{2} v+r_{h} \in U$. Then for all $w \in \mathbb{R}^{n}$,

$$
\begin{aligned}
& f\left(\bar{x}(t)+h y(t)+h^{2} w, \bar{u}(t)+h u(t)+h^{2} v+r_{h}\right) \\
& =f[t]+h\left(f_{x}[t] y(t)+f_{u}[t] u(t)\right)+h^{2}\left[f_{x}[t] w+f_{u}[t] v\right]+\frac{h^{2}}{2} f^{\prime \prime}[t] \xi(t) \xi(t)+o\left(h^{2}\right) .
\end{aligned}
$$

Since $\bar{u}(t)+h u(t)+h^{2} v+r_{h} \in U$, the left hand side of this equality belongs to the set $F\left(\bar{x}(t)+h y(t)+h^{2} w\right)$. From the definition of the second-order adjacent derivative of $F$ at the point $(\bar{x}(t), \dot{\bar{x}}(t), y(t), \dot{y}(t))$ in the direction $w$ we deduce that

$$
f_{x}[t] w+f_{u}[t] v+\frac{1}{2} f^{\prime \prime}[t] \xi(t) \xi(t) \in \mathrm{d}^{2} F(\bar{x}(t), \dot{\bar{x}}(t), y(t), \dot{y}(t))(w) .
$$

This and (4.2) imply that for a.e. $t \in[0,1]$ and for all $v \in T_{U}^{b(2)}(\bar{u}(t), u(t))$,

$$
f_{x}[t] w+f_{u}[t] v+\frac{1}{2} f^{\prime \prime}[t] \xi(t) \xi(t)+T_{F(\bar{x}(t))}(\dot{\bar{x}}(t)) \subset \mathrm{d}^{2} F[t](w) .
$$

Consequently, $\tilde{\mathcal{E}}^{2} \subset \mathcal{E}^{2}$. The control system (5.9) being linear in $w$, it is not difficult to deduce from the Aumann theorem, see for instance [1, p. 329], that the set $\tilde{\mathcal{E}}^{2}$ is convex. The Lemma is proved.

In general, the set $\tilde{\mathcal{E}}^{2}$ may be empty, for instance, when second order tangent sets are empty on a set of positive measure. Then (5.7) holds true, however not providing any information about $(\bar{x}, \bar{u})$.

We continue under the assumption of nonemptyness of $\tilde{\mathcal{E}}^{2}$. If $\xi(\cdot)=(y(\cdot), u(\cdot)) \in$ $\mathcal{C}_{0}(\bar{x}, \bar{u})$, then, by Lemmas 5.3 and 5.2, there exists $\lambda \in \Sigma_{+}^{k+1}$ satisfying (5.7). Fix controls $v(\cdot), \eta(\cdot)$ as in (5.10) and set

$$
\beta(t):=f_{u}[t] v(t)+\frac{1}{2} f^{\prime \prime}[t] \xi(t) \xi(t) .
$$

Let $p(\cdot)$ solve the adjoint system (2.5) with the transversality condition (2.6) and $w$ be the solution of (5.9). Then,

$$
\frac{\mathrm{d}}{\mathrm{d} t}(p(t) w(t))=-p(t) f_{x}[t] w(t)+p(t)\left(f_{x}[t] w(t)+\beta(t)+\eta(t)\right)=p(t)(\beta(t)+\eta(t)) .
$$


It follows that

$$
\int_{0}^{1} p(t)(\beta(t)+\eta(t)) \mathrm{d} t=p(1) w(1)-p(0) w(0)=l_{x}(\bar{x}(1), \lambda) w(1) .
$$

Consequently,

$$
\begin{aligned}
l_{x}(\bar{x}(1), \lambda) w(1) & =\int_{0}^{1} p(t)\left(f_{u}[t] v(t)+\frac{1}{2} f^{\prime \prime}[t] \xi(t) \xi(t)\right) \mathrm{d} t+\int_{0}^{1} p(t) \eta(t) \mathrm{d} t \\
& =\int_{0}^{1} \mathcal{H}_{u}[t] v(t) \mathrm{d} t+\frac{1}{2} \int_{0}^{1}\left\langle\mathcal{H}^{\prime \prime}[t] \xi(t), \xi(t)\right\rangle \mathrm{d} t+\int_{0}^{1} p(t) \eta(t) \mathrm{d} t .
\end{aligned}
$$

Recall that $\eta(\cdot)$ belongs to the convex cone

$$
K:=\left\{\eta(\cdot) \in L^{1}\left([0,1], \mathbb{R}^{n}\right): \eta(t) \in T_{F(\bar{x}(t))}(\dot{\bar{x}}(t)) \text { a.e. on }[0,1]\right\}
$$

and $v(\cdot) \in V^{2}(\bar{u}(\cdot), u(\cdot))$, see (3.7). Consequently, the second summand in (5.7) takes the form

$$
\begin{aligned}
\inf \left\langle l_{x}(\bar{x}(1), \lambda), \tilde{\mathcal{E}}^{2}\right\rangle & =\frac{1}{2} \int_{0}^{1}\left\langle\mathcal{H}^{\prime \prime}[t] \xi(t), \xi(t)\right\rangle \mathrm{d} t \\
& +\inf _{v \in V^{2}(\bar{u}, u)} \int_{0}^{1} \mathcal{H}_{u}[t] v(t) \mathrm{d} t+\inf _{\eta \in K} \int_{0}^{1} p(t) \eta(t) \mathrm{d} t .
\end{aligned}
$$

Then (5.7) implies

$$
\Omega(\xi, \lambda, p)+\inf _{v \in V^{2}(\bar{u}, u)} \int_{0}^{1} \mathcal{H}_{u}[t] v(t) \mathrm{d} t+\inf _{\eta \in K} \int_{0}^{1} p(t) \eta(t) \mathrm{d} t \geq 0,
$$

where $\Omega(\xi, \lambda, p)$ is defined by (3.9). Since $K$ is a cone, it follows that

$$
\Omega(\xi, \lambda, p)+\inf _{v \in V^{2}(\bar{u}, u)} \int_{0}^{1} \mathcal{H}_{u}[t] v(t) \mathrm{d} t \geq 0, \quad \int_{0}^{1} p(t) \eta(t) \mathrm{d} t \geq 0 \quad \forall \eta(\cdot) \in K .
$$

Let us analyze the last inequality. We claim that it implies

$$
p(t) \eta(t) \geq 0 \quad \text { a.e. } \quad \forall \eta(\cdot) \in K .
$$

Indeed, if there is $\eta(\cdot) \in K$ and a set $A \subset[0,1]$ of a positive measure such that $p(t) \eta(t)<0$ a.e. in $A$, then we can take $\tilde{\eta}(\cdot) \in K$ such that

$$
\tilde{\eta}(t)=\left\{\begin{aligned}
\eta(t) & \text { if } t \in A, \\
0 & \text { if not. }
\end{aligned}\right.
$$

Then $\int_{0}^{1} p(t) \tilde{\eta}(t) \mathrm{d} t<0$ leading to a contradiction. On the other hand, $f(\bar{x}(t), U)-$ $f[t] \subset T_{F(\bar{x}(t))}(\dot{\bar{x}}(t))$ a.e. Thus, by the measurable selection theorem,

$$
\inf _{v \in U} p(t)(f(\bar{x}(t), v)-f[t]) \geq 0 \quad \text { a.e., }
$$

that is

$$
\min _{v \in U} \mathcal{H}(\bar{x}(t), v, p(t))=\mathcal{H}(\bar{x}(t), \bar{u}(t), p(t)) \quad \text { a.e. }
$$

Hence $(\lambda, p(\cdot))$ satisfies the minimum principle (2.7) and

$$
\Omega(\xi, \lambda, p)+\inf _{v \in V^{2}(\bar{u}, u)} \int_{0}^{1} \mathcal{H}_{u}[t] v(t) \mathrm{d} t \geq 0 .
$$


Now, let us prove that, for given $u(\cdot), p(\cdot)$, the function $\gamma(t):=\Upsilon(u(t), p(t))$ is integrable on $[0,1]$ (see (3.8)). In the same way as in [1, Proof of Theorem 8.5.1] we show that $t \rightsquigarrow T_{U}^{b(2)}(\bar{u}(t), u(t))$ is a measurable set valued map with closed images. By [1, Theorem 8.2.8] also the set valued map

$$
t \rightsquigarrow \overline{H_{u}[t]\left(T_{U}^{b(2)}(\bar{u}(t), u(t))\right)}
$$

is measurable and has closed images (in the above the overline denotes the closure). This and [1, Theorem 8.2.11] imply that $t \mapsto \gamma(t):=\Upsilon(u(t), p(t))$ is measurable. To show that $\gamma$ is integrable, let us fix $\bar{v} \in V^{2}(\bar{u}, u)$. We use the representation $\gamma(t)=$ $\gamma^{+}(t)-\gamma^{-}(t)$, where $\gamma^{+}(t)=\max \{\gamma(t), 0\}$ and $\gamma^{-}(t)=\max \{-\gamma(t), 0\}, t \in[0,1]$. Since

$$
\gamma^{+}(t) \leq\left|H_{u}[t] \bar{v}(t)\right| \leq\|p(\cdot)\|_{\infty}\left|f_{u}[t] \bar{v}(t)\right| \quad \text { a.e. }
$$

the function $\gamma^{+}(\cdot)$ is integrable. It remains to show that $\gamma^{-}(t)$ is integrable.

We first prove that $\gamma(t)>-\infty$ for a.e. $t$. Indeed, otherwise there exists a set $A \subset[0,1]$ of positive measure such that for every $t \in A$ and every $j \geq 1$,

$$
Q_{j}(t):=\left\{w \in T_{U}^{b(2)}(\bar{u}(t), u(t)): \mathcal{H}_{u}[t] w \leq-j\right\} \neq \emptyset .
$$

Using [1, Theorem 8.2.9] we show that $Q_{j}: A \rightsquigarrow \mathbb{R}^{m}$ is measurable with closed images and, by the measurable selection theorem, there exists a measurable function $v_{j}: A \rightarrow \mathbb{R}^{m}$ such that $v_{j}(t) \in Q_{j}(t)$ for a.e. $t \in A$. For every integer $i \geq 1$, define $v_{j}^{i} \in V^{2}(\bar{u}, u)$ by

$$
v_{j}^{i}(t)= \begin{cases}v_{j}(t) & \text { if } t \in A \text { and }\left|f_{u}[t] v_{j}(t)\right| \leq i \\ \bar{v}(t) & \text { otherwise }\end{cases}
$$

and consider the increasing sequence of sets $A_{i}:=\left\{t \in A:\left|f_{u}[t] v_{j}(t)\right| \leq i\right\}$. Then $A=\cup_{i \geq 1} A_{i}$ and therefore for some $i$, we have meas $\left(A_{i}\right)>$ meas $(A) / 2$, where meas denotes the Lebesgue measure. Thus, by (5.12) applied to $v_{j}^{i}$, we have

$$
\Omega(\xi, \lambda, p)+\int_{0}^{1} H_{u}[t] v_{j}^{i}(t) \mathrm{d} t \geq 0
$$

Further,

$$
\int_{0}^{1} H_{u}[t] v_{j}^{i}(t) \mathrm{d} t=\int_{A_{i}} H_{u}[t] v_{j}(t) \mathrm{d} t+\int_{[0,1] \backslash A_{i}} H_{u}[t] \bar{v}(t) \mathrm{d} t .
$$

Moreover, by the definition of $v_{j}$,

$$
\int_{A_{i}} H_{u}[t] v_{j}(t) \mathrm{d} t \leq-j \operatorname{meas}\left(A_{i}\right)<-\frac{j}{2} \operatorname{meas}(A),
$$

and obviously, $\int_{[0,1] \backslash A_{i}} H_{u}[t] \bar{v}(t) \mathrm{d} t \leq \int_{0}^{1}\left|H_{u}[t] \bar{v}(t)\right| \mathrm{d} t$. Consequently,

$$
\Omega(\xi, \lambda, p)-\frac{j}{2} \operatorname{meas}(A)+\int_{0}^{1}\left|H_{u}[t] \bar{v}(t)\right| \mathrm{d} t \geq 0 .
$$


This leads to a contradiction whenever $j$ is sufficiently large and proves that $\gamma(t)$ is finite a.e.

Define next (we use below the same notation $Q_{j}$ for the new sets) for every integer $j \geq 1$ the set-valued map $Q_{j}:[0,1] \rightsquigarrow \mathbb{R}^{m}$ by

$$
Q_{j}(t)=\left\{w \in T_{U}^{b(2)}(\bar{u}(t), u(t)): \mathcal{H}_{u}[t] w \leq \gamma(t)+\frac{1}{j}\right\} .
$$

By [1, Theorem 8.2.9], $Q_{j}$ is measurable, with nonempty closed images and, by the measurable selection theorem, there exists a measurable function $\bar{v}_{j}:[0,1] \rightarrow \mathbb{R}^{m}$ such that $\bar{v}_{j}(t) \in Q_{j}(t)$ a.e. It follows that

$$
\gamma(t) \leq H_{u}[t] \bar{v}_{j}(t) \leq \gamma(t)+\frac{1}{j} \quad \text { a.e., } \quad j \geq 1 .
$$

Define for every integer $i \geq 1, \bar{v}^{i} \in V^{2}(\bar{u}, u)$ by

$$
\bar{v}^{i}(t)= \begin{cases}\bar{v}_{1}(t) & \text { if }\left|f_{u}(t) \bar{v}_{1}(t)\right| \leq i \\ \bar{v}(t) & \text { otherwise }\end{cases}
$$

By (5.12) applied to $\bar{v}^{i}$,

$$
\Omega(\xi, \lambda, p)+\int_{0}^{1} H_{u}[t] \bar{v}^{i}(t) \mathrm{d} t \geq 0
$$

and from the definition of $\bar{v}^{i}$, it follows that

$$
\int_{0}^{1} H_{u}[t] \bar{v}^{i}(t) \mathrm{d} t \leq \int_{0}^{1} \chi_{[0, i]}\left(\left|f_{u}[t] \bar{v}_{1}(t)\right|\right) H_{u}[t] \bar{v}_{1}(t) \mathrm{d} t+\int_{0}^{1}\left|H_{u}[t] \bar{v}(t)\right| \mathrm{d} t .
$$

Relations (5.13)-(5.15) imply that for all $i \geq 1$,

$$
\Omega(\xi, \lambda, p)+\int_{0}^{1} \chi_{[0, i]}\left(\left|f_{u}[t] \bar{v}_{1}(t)\right|\right) \gamma(t) \mathrm{d} t+1+\int_{0}^{1}\left|H_{u}[t] \bar{v}(t)\right| \mathrm{d} t \geq 0,
$$

where $\chi_{[0, i]}$ denotes the characteristic function of $[0, i]$. Substituting $\gamma(t)=\gamma^{+}(t)-$ $\gamma^{-}(t)$ in this inequality and taking into account that $\gamma^{+}(\cdot)$ is integrable, we obtain for all $i \geq 1$,

$$
\int_{0}^{1} \chi_{[0, i]}\left(\left|f_{u}[t] \bar{v}_{1}(t)\right|\right) \gamma^{-}(t) \mathrm{d} t \leq \Omega(\xi, \lambda, p)+\int_{0}^{1} \gamma^{+}(t) \mathrm{d} t+1+\int_{0}^{1}\left|H_{u}[t] \bar{v}(t)\right| \mathrm{d} t .
$$

Since $\cup_{i \geq 1}\left\{t \in[0,1]:\left|f_{u}(t) \bar{v}_{1}(t)\right| \leq i\right\}$ is the set of full measure in $[0,1]$, we deduce from the last inequality that $\gamma^{-}(\cdot)$ is integrable, and thus, $\gamma(\cdot)$ is integrable.

From (5.13) and the integrability of $\gamma$ it follows that $H_{u}[\cdot] \bar{v}_{j}(\cdot)$ is integrable for every $j \geq 1$. Fix $j \geq 1$. For every integer $i \geq 1$ consider the set $B_{i}=\{t \in[0,1]$ : $\left.\left|f_{u}[t] \bar{v}_{j}(t)\right| \leq i\right\}$ and define $\bar{v}_{j}^{i} \in V^{2}(\bar{u}, u)$ by

$$
\bar{v}_{j}^{i}(t)= \begin{cases}\bar{v}_{j}(t) & \text { if } t \in B_{i} \\ \bar{v}(t) & \text { otherwise. }\end{cases}
$$

Then, for every $i$,

$$
\begin{gathered}
\Omega(\xi, \lambda, p)+\int_{0}^{1} \gamma(t) \mathrm{d} t+\frac{1}{j} \geq \Omega(\xi, \lambda, p)+\int_{0}^{1} \mathcal{H}_{u}[t] \bar{v}_{j}(t) \mathrm{d} t= \\
\Omega(\xi, \lambda, p)+\int_{B_{i}} \mathcal{H}_{u}[t] \bar{v}_{j}^{i}(t) \mathrm{d} t+\int_{[0,1] \backslash B_{i}} \mathcal{H}_{u}[t] \bar{v}_{j}(t) \mathrm{d} t .
\end{gathered}
$$


On the other hand, by (5.12), applied to $\bar{v}_{j}^{i}$

$$
\begin{aligned}
\Omega(\xi, \lambda, p) & +\int_{B_{i}} \mathcal{H}_{u}[t] \bar{v}_{j}^{i}(t) \mathrm{d} t=\Omega(\xi, \lambda, p)+\int_{0}^{1} \mathcal{H}_{u}[t] \bar{v}_{j}^{i}(t) \mathrm{d} t-\int_{[0,1] \backslash B_{i}} \mathcal{H}_{u}[t] \bar{v}(t) \mathrm{d} t \\
& \geq-\int_{[0,1] \backslash B_{i}}\left|\mathcal{H}_{u}[t] \bar{v}(t)\right| \mathrm{d} t .
\end{aligned}
$$

Consequently, for every integer $i \geq 1$,

$$
\Omega(\xi, \lambda, p)+\int_{0}^{1} \gamma(t) \mathrm{d} t+\frac{1}{j} \geq \int_{[0,1] \backslash B_{i}} \mathcal{H}_{u}[t] \bar{v}_{j}(t) \mathrm{d} t-\int_{[0,1] \backslash B_{i}}\left|\mathcal{H}_{u}[t] \bar{v}(t)\right| \mathrm{d} t .
$$

Since meas $\left([0,1] \backslash B_{i}\right) \rightarrow 0$ when $i \rightarrow \infty$, taking the limit, we deduce that

$$
\Omega(\xi, \lambda, p)+\int_{0}^{1} \gamma(t) \mathrm{d} t+\frac{1}{j} \geq 0 .
$$

The integer $j \geq 1$ being arbitrary, inequality (3.10) follows. Theorem 3.2 is completely proved.

Proof of Theorem 3.4 It is enough to observe that $F(x)=f_{0}(x)+G(x) \operatorname{co} U$. Then the same proof implies the result.

6. Some special cases of $U$. In this section we consider problem (2.1)-(2.3) with the set $U$ having a special structure. In all the considered cases, the main result will be simplified.

6.1. The case when $U$ is a convex polytope. Suppose that $U$ is a convex polytope, i.e., a compact set, being an intersection of a finite number of affine halfspaces. Let $u_{0} \in U$. If $u_{0} \in \operatorname{int} U$, then, obviously, $T_{U}^{b}\left(u_{0}\right)=\mathbb{R}^{m}$ and $T_{U}^{b(2)}\left(u_{0}, u\right)=$ $\mathbb{R}^{m}$ for all $u \in \mathbb{R}^{m}$.

If $u_{0} \in \partial U$, then $T_{U}^{b}\left(u_{0}\right)=\operatorname{cone}\left(U-u_{0}\right)$ is a finite faced convex cone, which coincides with $U-u_{0}$ in a small neighbourhood of zero. Further, if $u \in \operatorname{int} T_{U}^{b}\left(u_{0}\right)$, then $T_{U}^{b(2)}\left(u_{0}, u\right)=\mathbb{R}^{m}$. If $u \in \partial T_{U}^{b}\left(u_{0}\right)$, then $T_{U}^{b(2)}\left(u_{0}, u\right)$ is a finite faced convex cone, $T_{U}^{b(2)}\left(u_{0}, u\right)=\operatorname{cone}\left(T_{U}^{b}\left(u_{0}\right)-u\right)$. Note, that, in any case, $0 \in T_{U}^{b(2)}\left(u_{0}, u\right)$.

Suppose that a $C^{2}$ function $\psi: \mathbb{R}^{m} \rightarrow \mathbb{R}$ attains its minimum on $U$ at a point $u_{0} \in U$. Then, as we know (see Section 1),

$$
\psi^{\prime}\left(u_{0}\right) u \geq 0 \quad \forall u \in T_{U}^{b}\left(u_{0}\right)
$$

and, moreover, for any $u \in T_{U}^{b}\left(u_{0}\right)$ such that $\psi^{\prime}\left(u_{0}\right) u=0$ the following is true:

$$
\psi^{\prime}\left(u_{0}\right) v+\frac{1}{2}\left\langle\psi^{\prime \prime}\left(u_{0}\right) u, u\right\rangle \geq 0 \quad \forall v \in T_{U}^{b(2)}\left(u_{0}, u\right) .
$$

Since $T_{U}^{b(2)}\left(u_{0}, u\right)$ is a nonempty closed cone, this condition is equivalent to

$$
\left\langle\psi^{\prime \prime}\left(u_{0}\right) u, u\right\rangle \geq 0, \quad \psi^{\prime}\left(u_{0}\right) v \geq 0 \quad \forall v \in T_{U}^{b(2)}\left(u_{0}, u\right) .
$$

Now, consider problem (2.1)-(2.3) with the set $U$ being a convex polytope. Denote by ext $U$ the set of all vertices (extremal points) of $U$. Let an admissible pair $(\bar{x}, \bar{u})$ affords a strong local minimum in this problem and assume that

$$
\bar{u}(t) \in \operatorname{ext} U \quad \text { a.e. in } \quad[0,1] .
$$


As it follows from the minimum principle (2.7), this assumption is quite natural in the case when the control system is affine in the control.

Under assumption (6.2), condition (3.5) is automatically fulfilled, and then

$$
\mathcal{C}_{0}(\bar{x}, \bar{u})=\mathcal{C}(\bar{x}, \bar{u})
$$

Let $\xi(\cdot)=(y(\cdot), u(\cdot)) \in \mathcal{C}(\bar{x}, \bar{u})$. Then condition (3.10) holds with some $(\lambda, p(\cdot)) \in$ $M(\bar{x}, \bar{u})$.

Consider the function $\Upsilon(u(\cdot), p(\cdot))$ appearing in the necessary condition (3.10). We know that $u(t) \in T_{U}^{b}(\bar{u}(t))$ a.e., and by Lemma $3.1, \mathcal{H}_{u}[t] u(t)=0$ a.e. Take any $t \in[0,1]$ such that both conditions hold and let $v \in T_{U}^{b(2)}(\bar{u}(t), u(t))$. In view of (6.1), $\mathcal{H}_{u}[t] v \geq 0$ a.e. Since $0 \in T_{U}^{b(2)}(\bar{u}(t), u(t))$, we obtain $\Upsilon(u(t), p(t))=0$ a.e.

We have proved the following theorem.

TheOrem 6.1. Let $U$ be a convex polytope, and let $(\bar{x}, \bar{u})$ be a strong local minimizer of problem (2.1)-(2.3) with $\bar{u}$ satisfying condition (6.2). Then, for any $\xi(\cdot)=(y(\cdot), u(\cdot)) \in \mathcal{C}(\bar{x}, \bar{u})$, there exists $(\lambda, p(\cdot)) \in M(\bar{x}, \bar{u})$, such that

$$
\left\langle l_{x x}(\bar{x}(1), \lambda) y(1), y(1)\right\rangle+\int_{0}^{1}\left\langle\mathcal{H}^{\prime \prime}[t] \xi(t), \xi(t)\right\rangle \mathrm{d} t \geq 0 .
$$

6.2. Two additional examples of control set $U$. The second-order necessary condition of Theorem 3.2 can be useful in many cases, where the known conditions cannot be applied. By known conditions, we mean conditions, obtained for the case, when $U$ is given by a system of smooth inequalities with positively (or even linearly) independent gradients of active control constraints (see also Sections 7 and 8 below). Let us give two such "exotic" examples.

1. In the space $\mathbb{R}^{m}$, consider the "reper"

$$
U=\bigcup_{i=1}^{m}\left\{[0,1] e_{i}\right\},
$$

where $e_{i}=(0, \ldots, 0,1,0, \ldots, 0)$ ( 1 is at the $i$ th position).

Obviously, if $u_{0} \in(0,1) e_{i}$ for some $i$, then $T_{U}^{b}\left(u_{0}\right)=\mathbb{R} e_{i}$, and $T_{U}^{b}\left(e_{i}\right)=\mathbb{R}_{-} e_{i}$ for all $i$, and $T_{U}^{b}(0)=\bigcup_{i=1}^{m} \mathbb{R}_{+} e_{i}$. Further, for every $u_{0} \in U$ and every $0 \neq u \in T_{U}^{b}\left(u_{0}\right)$ we have $T_{U}^{b(2)}\left(u_{0}, u\right)=\mathbb{R} u$, and $T_{U}^{b(2)}\left(u_{0}, 0\right)=T_{U}^{b}\left(u_{0}\right)$. Consequently, $0 \in T_{U}^{b(2)}\left(u_{0}, u\right)$ for any $u_{0} \in U$ and any $u \in T_{U}^{b}\left(u_{0}\right)$.

Let $(\bar{x}(\cdot), \bar{u}(\cdot))$ be a point of a strong local minimum in problem (2.1)-(2.3), where $U$ is the reper $(6.5)$ and $\bar{u}(t) \in\left\{0, e_{1}, \ldots, e_{m}\right\}$ a.e. Then condition (6.3) holds. Similarly to the previous subsection, we can prove that for any $\xi(\cdot)=(y(\cdot), u(\cdot)) \in \mathcal{C}(\bar{x}, \bar{u})$, there exists $(\lambda, p(\cdot)) \in M(\bar{x}, \bar{u})$ satisfying $(6.4)$.

2. Let $U$ be the union of two balls in $\mathbb{R}^{m}$ with exactly one common point $\hat{u}$. This case is also covered by Theorem 3.2. Note that in this case the normals to each ball at the point $\hat{u}$ are even positively dependent.

Obviously, the row of such "exotic" examples can be continued. 
7. The case when $U$ is given by inequalities. Consider now problem (2.1)(2.3), when the set $U$ is defined by the system of inequalities

$$
U=\left\{u \in \mathbb{R}^{m}: \varphi_{i}(u) \leq 0, \quad i=1, \ldots, q\right\} .
$$

We assume that $\varphi_{i}: \mathbb{R}^{m} \rightarrow \mathbb{R}$ are $C^{2}$ functions, that $U$ is compact and at each point $u \in \partial U$ the gradients $\varphi_{i}^{\prime}(u), i \in I_{\varphi}(u)$ are positively independent, where $I_{\varphi}(u)=\{i$ : $\left.\varphi_{i}(u)=0\right\}$ is the set of active indices at $u$ and $\varphi=\left(\varphi_{1}, \ldots, \varphi_{q}\right)$. Recall that vectors $a_{1}, \ldots, a_{s} \in \mathbb{R}^{m}$ are said to be positively independent if conditions $\sum_{i} \lambda_{i} a_{i}=0, \lambda_{i} \geq$ $0, i=1, \ldots, s$ imply that all $\lambda_{i}$ are equal to zero, or equivalently, if $0 \notin \operatorname{co}\left\{a_{1} \ldots, a_{s}\right\}$. By the separation theorem, the latter means that there exists $v \in \mathbb{R}^{m}$ such that $\left\langle a_{i}, v\right\rangle\langle 0, i=1, \ldots, s$. The converse is also true: if this condition is fulfilled, then the vectors $a_{1}, \ldots, a_{s}$ are positively independent. Then, according to [1, Chapter 4], for every $u_{0} \in U$,

$$
T_{U}^{b}\left(u_{0}\right)=\left\{u \in \mathbb{R}^{m}: \varphi_{i}^{\prime}\left(u_{0}\right) u \leq 0, i \in I_{\varphi}\left(u_{0}\right)\right\} .
$$

Let $(\bar{x}, \bar{u}) \in \Xi$ be a strong local minimizer in problem (2.1)-(2.3), and hence the minimum principle (2.4)-(2.7) holds. From the minimum condition (2.7) it follows that there exists a measurable vector function $\mu(\cdot)=\left(\mu_{1}(\cdot), \ldots, \mu_{q}(\cdot)\right)$ such that

$$
\begin{gathered}
\mu_{1}(t) \geq 0, \ldots, \mu_{q}(t) \geq 0, \quad \mu_{i}(t) \varphi_{i}(\bar{u}(t))=0, \quad \text { a.e. }, \quad i=1, \ldots, q, \\
\mathcal{H}_{u}(\bar{x}(t), \bar{u}(t), p(t))+\sum_{i=1}^{q} \mu_{i}(t) \varphi_{i}^{\prime}(\bar{u}(t))=0 \quad \text { a.e. }
\end{gathered}
$$

(see $[6$, Section 5] for details.)

In general such $\mu$ is not uniquely defined. Due to the positive independence assumption for gradients $\varphi_{i}^{\prime}$ it can be deduced that $\mu(\cdot)$ can be chosen so that it is essentially bounded (see, for instance, [6, Section 5]). In the case of linear independence assumption of gradients $\left\{\varphi_{i}^{\prime}(u)\right\}_{i \in I_{\varphi}(u)}$ for all $u \in \partial U, \mu(\cdot)$ is uniquely defined by (7.3) and hence it is uniquely defined by $\lambda$.

Let us introduce the augmented Hamiltonian

$$
\mathcal{H}^{a}(x, u, p, \mu)=\mathcal{H}(x, u, p)+\mu \varphi(u),
$$

where $\varphi=\left(\varphi_{1}, \ldots, \varphi_{q}\right), \mu \in \mathbb{R}^{q *}$. Then condition (7.3) can be written as

$$
\mathcal{H}_{u}^{a}(\bar{x}(t), \bar{u}(t), p(t), \mu(t))=0 \quad \text { a.e. }
$$

This is a stationarity condition for the Hamiltonian, with respect to the control variable, following from the minimum principle. Let us introduce the set

$$
\begin{aligned}
M^{a}(\bar{x}, \bar{u})=\{(\lambda, p(\cdot), \mu(\cdot)): & (\lambda, p(\cdot)) \in M(\bar{x}, \bar{u}), \mu(\cdot) \in L^{\infty}\left([0,1], \mathbb{R}^{q}\right), \\
& \text { and conditions (7.2), (7.3) hold. }\}
\end{aligned}
$$

Now let us turn to the second-order conditions. Consider the critical cone $\mathcal{C}(\bar{x}, \bar{u})$ and set

$$
\mathcal{M}_{i 0}=\left\{t \in[0,1]: \bar{u}(t) \in \partial U, \varphi_{i}(\bar{u}(t))=0\right\}, \quad i=1, \ldots, q .
$$


Condition (3.3) is equivalent to

$$
\varphi_{i}^{\prime}(\bar{u}(t)) u(t) \leq 0 \quad \text { a.e. in } \quad \mathcal{M}_{i 0}, \quad i=1, \ldots, q .
$$

Thus, in the considered case, the following proposition holds.

Proposition 7.1. The critical cone $\mathcal{C}(\bar{x}, \bar{u})$ is defined by conditions (3.1), (3.2), and (7.4).

For any $\delta>0$, set

$$
\mathcal{M}_{i \delta}=\left\{t \in[0,1]: \operatorname{dist}_{\partial U}(\bar{u}(t)) \leq \delta, \varphi_{i}(\bar{u}(t)) \geq-\delta\right\}, \quad i=1, \ldots, q .
$$

Consider $u(\cdot)$ satisfying a stronger condition than (7.4): there exists a $\delta>0$ such that

$$
\varphi_{i}^{\prime}(\bar{u}(t)) u(t) \leq 0 \quad \text { a.e. on } \quad \mathcal{M}_{i \delta}, \quad i=1, \ldots, q .
$$

Lemma 7.2. Let $u(\cdot)$ satisfies condition (7.5) with some $\delta>0$. Then condition (3.5) also holds with some constants $h_{0}>0, c>0$ (i.e., with function $c(\cdot)$ in (3.5) independent from time).

To prove this lemma we need the two auxiliary results below. For any real $a$, we will use the representation $a=a^{+}-a^{-}$, where $a^{+}=\max \{a, 0\}$ and $a^{-}=\max \{-a, 0\}$. Similarly, for any vector $b \in \mathbb{R}^{m}$ we write $b=b^{+}-b^{-}$, where $b^{+}=\left(b_{1}^{+}, \ldots, b_{m}^{+}\right)$, $b^{-}=\left(b_{1}^{-}, \ldots, b_{m}^{-}\right)$. Observe that if $u \in \partial U$ then $I_{\varphi}(u) \neq \emptyset$. Under the assumptions made about the functions $\varphi_{i}$, the following local estimate for the distance to the set $U$ holds.

LEMma 7.3. Let $u_{0} \in \partial U$. Then there exist a neighbourhood $\mathcal{V}$ of $u_{0}$ and a constant $c>0$ such that for any $u \in \mathcal{V}$ we can find $u^{\prime} \in U$ satisfying

$$
\left|u-u^{\prime}\right| \leq c \sum_{i=1}^{q} \varphi_{i}^{+}(u), \quad \text { where } \varphi_{i}^{+}:=\max \left\{\varphi_{i}, 0\right\} .
$$

Proof This estimate is well-known under the assumption of linear independence of the gradients $\varphi_{i}^{\prime}\left(u_{0}\right), i \in I_{\varphi}\left(u_{0}\right)$ (it follows from the Lusternik-Graves theorem). In order to prove it under the assumption of positive independence of these gradients, we will use [4, Theorem 3.1]. Let $B$ stands for the closed unit ball in $\mathbb{R}^{q}$ (we use the Euclidean norm in $\mathbb{R}^{q}$ and in $\mathbb{R}^{m}$ ). From the assumption of positive independence of gradients $\varphi_{i}^{\prime}\left(u_{0}\right), i \in I_{\varphi}\left(u_{0}\right)$ and the continuity of the gradients it follows that there exist a vector $v_{0} \in B$, a neighbourhood $B_{\varepsilon}\left(u_{0}\right)(\varepsilon>0)$ of the point $u_{0}$, and a constant $\rho>0$ such that for all $w \in B_{\varepsilon}\left(u_{0}\right)$,

$$
\left\langle\varphi_{i}^{\prime}(w), v_{0}\right\rangle<-\rho, \quad\left|\left\langle\varphi_{i}^{\prime}(w), v_{0}\right\rangle\right|+\rho<\frac{1}{\sqrt{q}}, \quad i \in I_{\varphi}\left(u_{0}\right) .
$$

Hence $\rho \leq 1$. Without loss of generality we may assume that $I_{\varphi}\left(u_{0}\right)=\{1, \ldots, q\}$, i.e., all indices are active at $u_{0}$. Set

$$
\alpha_{i}:=-\left\langle\varphi_{i}^{\prime}(w), v_{0}\right\rangle-\rho>0, \quad \forall i=1, \ldots, q .
$$

Then, in view of the second relation in $(7.7), \alpha_{i} \in\left[0, \frac{1}{\sqrt{q}}\right], i=1, \ldots, q$. Since $\left\langle\varphi_{i}^{\prime}(w), v_{0}\right\rangle<0$,

$$
\beta_{i}:=-\left\langle\varphi_{i}^{\prime}(w), v_{0}\right\rangle>0, \quad i=1, \ldots, q
$$


Again, in view of the second relation in (7.7), $\beta_{i}+\rho \in\left[0, \frac{1}{\sqrt{q}}\right], i=1, \ldots, q$.

Let $\theta=\left(\theta_{1}, \ldots, \theta_{q}\right) \in \rho B$ be an arbitrary point. Then there exist $\gamma_{i} \in[0,1]$ such that $\left(1-\gamma_{i}\right)(-\rho)+\gamma_{i} \rho=\theta_{i}, i=1, \ldots, q$. From (7.8) and (7.9) we deduce that

$$
-\rho=\left\langle\varphi_{i}^{\prime}(w), v_{0}\right\rangle+\alpha_{i}, \quad \rho=\left\langle\varphi_{i}^{\prime}(w), v_{0}\right\rangle+\beta_{i}+\rho, \quad i=1, \ldots, q .
$$

Multiplying the first equality by $\left(1-\gamma_{i}\right)$ and the second one by $\gamma_{i}$ and adding the results we get

$$
\theta_{i}=\left\langle\varphi_{i}^{\prime}(w), v_{0}\right\rangle+\zeta_{i}, \quad i=1, \ldots, q,
$$

where $\zeta_{i}=\left(1-\gamma_{i}\right) \alpha_{i}+\gamma_{i}\left(\beta_{i}+\rho\right) \geq 0$. Since $\alpha_{i} \in\left[0, \frac{1}{\sqrt{q}}\right]$ and $\beta_{i}+\rho \in\left[0, \frac{1}{\sqrt{q}}\right]$, it follows that $\zeta_{i} \in\left[0, \frac{1}{\sqrt{q}}\right]$ and hence $\theta \in\left\langle\varphi^{\prime}(w), v_{0}\right\rangle+\left(\mathbb{R}_{+}^{q} \cap B\right)$. Therefore,

$$
\rho B \subset\left\langle\varphi^{\prime}(w), v_{0}\right\rangle+\left(\mathbb{R}_{+}^{q} \cap B\right) .
$$

Consider the metric space $\mathbb{R}^{m} \times \mathbb{R}_{+}^{q}$ with the distance

$$
d\left((u, r),\left(u^{\prime}, r^{\prime}\right)\right)=\max \left\{\left|u-u^{\prime}\right|,\left|r-r^{\prime}\right|\right\}
$$

and define the mapping $G: \mathbb{R}^{m} \times \mathbb{R}_{+}^{q} \rightarrow \mathbb{R}^{q}$ by $G(w, r):=\varphi(w)+r$. For every $w \in \mathbb{R}^{m}, r \in \mathbb{R}_{+}^{q}$ set

$$
G^{(1)}(w, r):=\operatorname{Limsup}_{h \rightarrow 0+} \frac{G\left(B_{h}(w, r)\right)-G(w, r)}{h},
$$

where $B_{h}(w, r)$ denotes the closed ball in $\mathbb{R}^{m} \times \mathbb{R}_{+}^{q}$ centered at $(w, r)$ of radius $h>0$ and Limsup stands for the Kuratowski upper limit.

Then for every $w \in B_{\varepsilon}\left(u_{0}\right)$ and all $r \in \mathbb{R}_{+}^{m}$,

$$
\varphi^{\prime}(w) v_{0}+\left(\mathbb{R}_{+}^{q} \cap B\right) \subset G^{(1)}(w, r) .
$$

This and (7.10) imply

$$
\rho B \subset G^{(1)}(w, r) \quad \forall w \in B_{\varepsilon}\left(u_{0}\right), \forall r \in \mathbb{R}_{+}^{q} .
$$

Hence, condition (11) of Theorem 3.1 in [4] holds true for the mapping $G$ at $\left(u_{0}, r\right)$ with $r \in \mathbb{R}_{+}^{q}$.

Observe that for any $u \in \mathbb{R}^{m}$,

$$
G(u, 0)-\left(-\varphi^{-}(u)\right)=\varphi(u)+\varphi^{-}(u)=\varphi^{+}(u)=\varphi^{+}(u)-\varphi^{+}\left(u_{0}\right) .
$$

Let $L>0$ be a Lipschitz constant of the function $\varphi^{+}(\cdot)$ in the neighbourhood $B_{\frac{\varepsilon}{4}}\left(u_{0}\right)$ of $u_{0}$. Then

$$
\left|G(u, 0)-\left(-\varphi^{-}(u)\right)\right|=\left|\varphi^{+}(u)-\varphi^{+}\left(u_{0}\right)\right| \leq L\left|u-u_{0}\right| \quad \forall u \in B_{\frac{\varepsilon}{4}}\left(u_{0}\right) .
$$

Applying [4, Theorem 3.1] we deduce that if $u \in B_{\frac{\varepsilon}{4}}\left(u_{0}\right)$ and $L\left|u-u_{0}\right|<\frac{\varepsilon \rho}{8}$, i.e. $u$ belongs to a small enough neighbourhood of $u_{0}$, then there exists $\left(u^{\prime}, r^{\prime}\right) \in \mathbb{R}^{m} \times \mathbb{R}_{+}^{q}$ satisfying

$$
G\left(u^{\prime}, r^{\prime}\right)=-\varphi^{-}(u), \quad\left|u^{\prime}-u\right| \leq \frac{2}{\rho}\left|\varphi(u)-\left(-\varphi^{-}(u)\right)\right| .
$$


The first relation implies $\varphi\left(u^{\prime}\right)+r^{\prime}=-\varphi^{-}(u) \leq 0$, whence $\varphi\left(u^{\prime}\right) \leq 0$, i.e. $u^{\prime} \in U$. From the second relation we obtain $\left|u^{\prime}-u\right| \leq \frac{2}{\rho}\left|\varphi^{+}(u)\right|$. The lemma is proved.

COROLlary 7.4. There exist a neighbourhood $\mathcal{W}$ of the boundary $\partial U$ and a constant $c>0$ such that for any $u \in \mathcal{W}$ we can find $u^{\prime} \in U$ such that estimate (7.6) holds.

Proof Let $u_{0} \in \partial U$. Then there exist a neighbourhood $\mathcal{V}\left(u_{0}\right)$ of $u_{0}$ and a constant $c\left(u_{0}\right)>0$ such that for any $u \in \mathcal{V}\left(u_{0}\right)$ we can find $u^{\prime} \in U$ satisfying (7.6) with $c$ replaced by $c\left(u_{0}\right)$. The neighbourhoods $\mathcal{V}\left(u_{0}\right), u_{0} \in \partial U$ form a covering of the compact set $\partial U$. Let $\mathcal{V}\left(u_{1}\right), \ldots, \mathcal{V}\left(u_{s}\right)$ be a finite subcovering of $\partial U$. Take $\mathcal{W}=\mathcal{V}\left(u_{1}\right) \cup \ldots \cup \mathcal{V}\left(u_{s}\right), c=\min \left\{c\left(u_{1}\right), \ldots, c\left(u_{s}\right)\right\}$. Then for any $u \in \mathcal{W}$ there exists $u^{\prime} \in U$ satisfying (7.6).

Proof of Lemma 7.2 For every $i=1, \ldots, q$ and a.e. $t \in[0,1]$, we have

$$
\varphi_{i}(\bar{u}(t)+h u(t))=\varphi_{i}(\bar{u}(t))+h \varphi_{i}^{\prime}(\bar{u}(t)) u(t)+\frac{h^{2}}{2}\left\langle\varphi_{i}^{\prime \prime}(\bar{u}(t)) u(t), u(t)\right\rangle+h^{2} r_{h}(t),
$$

where $\left\|r_{h}(\cdot)\right\|_{\infty} \rightarrow 0$ as $h \rightarrow 0+$. This and (7.5) imply that there exists $c_{1} \geq 0$ such that

$$
\varphi_{i}(\bar{u}(t)+h u(t)) \leq c_{1} h^{2} \quad \forall h \in[0,1], \quad \text { a.e. on } \quad \mathcal{M}_{i \delta}, \quad i=1, \ldots, q, .
$$

Since for a.e. $t \in[0,1] \backslash \mathcal{M}_{i \delta}$ we have $\varphi_{i}(\bar{u}(t)+h u(t)) \leq 0$ for all $h>0$ small enough and $i=1, \ldots, q$, it follows that

$$
\sum_{i=1}^{q} \varphi_{i}^{+}(\bar{u}(t)+h u(t)) \leq c_{1} h^{2} \quad \text { a.e. in } \quad[0,1]
$$

for all $h>0$ small enough. By Corollary 7.4, this implies that estimate (3.5) holds with $c(t)=c c_{1}>0$ and some $h_{0}>0$. The lemma is proved.

Now consider the function $\Upsilon(u(\cdot), p(\cdot))$ in the necessary condition (3.10). It was proved in $[6$, Section 5], that $\mu(\cdot)$ can be chosen in such a way that it is essentially bounded and

$$
\Upsilon(u(t), p(t))=\frac{1}{2} \sum_{i=1}^{q} \mu_{i}(t)\left\langle\varphi_{i}^{\prime \prime}(\bar{u}(t)) u(t), u(t)\right\rangle \quad \text { a.e. }
$$

and moreover, conditions (7.2) and (7.3) hold. Define

$$
\Omega^{a}(\xi, \lambda, p, \mu):=\frac{1}{2}\left\langle l_{x x}(\bar{x}(1), \lambda) y(1), y(1)\right\rangle+\frac{1}{2} \int_{0}^{1}\left\langle\mathcal{H}^{a \prime \prime}[t] \xi(t), \xi(t)\right\rangle \mathrm{d} t .
$$

Applying now Theorem 3.2 we obtain the following result.

TheOREM 7.5. Let $(\bar{x}, \bar{u})$ be a strong local minimizer. Then $M^{a}(\bar{x}, \bar{u}) \neq \emptyset$ and for any $\xi(\cdot)=(y(\cdot), u(\cdot)) \in \mathcal{C}(\bar{x}, \bar{u})$ satisfying (7.5) with some $\delta>0$ (depending on $\xi$ ), there exists $(\lambda, p, \mu) \in M^{a}(\bar{x}, \bar{u})$ such that $\Omega^{a}(\xi, \lambda, p, \mu) \geq 0$.

Under a stronger assumption, the conclusion of the above theorem can be strengthened. This will be done in the next section. 
8. The case of linear independence of the gradients of control constraints. We continue to consider problem (2.1)-(2.3) with $U$ given by the system of inequalities (7.1). We again assume $C^{2}$ smoothness of $\varphi_{i}$, but now, instead of positive independence of the gradients $\varphi_{i}^{\prime}$ of active control constraints, we suppose that these gradients are linearly independent on $\partial U$. Then Theorem 7.5 can be refined: the assumption that $\xi=(y, u)$ satisfies (7.5) with some $\delta>0$ in this theorem can be omitted. So, we will prove the following theorem, using some arguments from [10].

THEOREM 8.1. If $(\bar{x}, \bar{u})$ is a point of a strong local minimum, then the set $M^{a}(\bar{x}, \bar{u})$ is nonempty and for any $\xi=(y, u) \in \mathcal{C}(\bar{x}, \bar{u})$ there exists $(\lambda, p, \mu) \in M^{a}(\bar{x}, \bar{u})$ such that $\Omega^{a}(\xi, \lambda, p, \mu) \geq 0$. Moreover $\mu$ is uniquely defined by $\lambda$.

In order to prove this theorem, consider a linear operator

$$
T: \Xi \ni(y(\cdot), u(\cdot)) \mapsto\left(g^{\prime}(\bar{x}(1)) y(1) \in \mathbb{R}^{k+1},\right.
$$

where $g=\left(g_{0}, g_{1}, \ldots, g_{k}\right)$ and observe that, $|T(y, u)| \leq c_{g}|y(1)|$ for all $\xi=(y, u) \in \Xi$, with some constant $c_{g}>0$ independent from $\xi$. For $\delta \geq 0$, define

$$
\begin{aligned}
\Gamma_{\delta}= & \{(y(\cdot), u(\cdot)) \in \Xi: y \text { solves }(3.2) \text { and } \\
& \left.\varphi_{i}^{\prime}(\bar{u}(t)) u(t)=0 \text { a.e. in } \mathcal{M}_{i \delta}, \quad i=1, \ldots, q\right\} .
\end{aligned}
$$

Note that $\left\{\Gamma_{\delta}\right\}$ is a decreasing family of subspaces of $\Xi$, and $\Gamma_{\delta} \subset \Gamma_{0}$ for all $\delta>0$, where $\Gamma_{0}$ is defined by system (8.1) with the last condition of the form

$$
\varphi_{i}^{\prime}(\bar{u}(t)) u(t)=0 \text { a.e. in } \mathcal{M}_{i 0}, \quad i=1, \ldots, q .
$$

Lemma 8.2. There exists a $\delta_{1}>0$ such that $T\left(\Gamma_{0}\right)=T\left(\Gamma_{\delta_{1}}\right)$.

Proof Let $b_{1}, \ldots, b_{s}$ be a basis in $T\left(\Gamma_{0}\right)$, and let $\left(y_{1}, u_{1}\right), \ldots,\left(y_{s}, u_{s}\right) \in \Gamma_{0}$ be such that $T\left(y_{i}, u_{i}\right)=b_{i}, i=1, \ldots, s$. Define

$$
m_{j \delta}=\mathcal{M}_{j \delta} \backslash \mathcal{M}_{j 0}, \quad j=1, \ldots, q, \quad m_{\delta}=\cup_{j=1}^{q} m_{j \delta} .
$$

The Lebesgue measure of $m_{\delta}$ goes to zero when $\delta \rightarrow 0+$. Set $u_{i \delta}(t)=u_{i}(t) \chi_{[0,1] \backslash m_{\delta}}(t)$, $i=1, \ldots, s$, where $\chi_{[0,1] \backslash m_{\delta}}(t)$ is the characteristic function of the set $[0,1] \backslash m_{\delta}$, and let $y_{i \delta}$ be the corresponding solution to the system

$$
\dot{y}(t)=f_{x}[t] y(t)+f_{u}[t] u_{i \delta}(t), \quad y(0)=0 .
$$

Obviously, $T\left(y_{i \delta}, u_{i \delta}\right) \rightarrow b_{i}$, as $\delta \rightarrow 0+$, for $i=1, \ldots, s$. Hence, for all $\delta>0$ small enough, the vectors $T\left(y_{i \delta}, u_{i \delta}\right), i=1, \ldots, s$ are linearly independent. Since $\left(y_{i \delta}, u_{i \delta}\right) \in$ $\Gamma_{\delta} \subset \Gamma_{0}, i=1, \ldots, s$, the assertion of the lemma follows.

Let $\xi(\cdot)=(x(\cdot), u(\cdot)) \in \mathcal{C}(\bar{x}, \bar{u})$. Since the gradients $\varphi_{i}^{\prime}(\cdot)$ of active control constraints are linearly independent on $\partial U$, they are linearly independent in a small neighbourhood of $\partial U$. Hence there exists $c>0$ such that for every $\delta>0$ small enough we can find $u^{\delta} \in L^{\infty}\left([0,1], \mathbb{R}^{m}\right)$ satisfying the following conditions

$$
\begin{gathered}
\varphi_{i}^{\prime}(\bar{u}(t)) u^{\delta}(t)=\varphi_{i}^{\prime}(\bar{u}(t)) u(t) \quad \text { a.e. in } m_{i \delta}, \\
\varphi_{i}^{\prime}(\bar{u}(t)) u^{\delta}(t)=0 \quad \text { a.e. on } \mathcal{M}_{i 0}, \quad i=1, \ldots, q,
\end{gathered}
$$




$$
\left\{t \in[0,1]: u^{\delta}(t) \neq 0\right\} \subset m_{\delta}, \quad\left\|u^{\delta}\right\|_{\infty} \leq c\|u\|_{\infty} .
$$

Consequently,

$$
\left\|u^{\delta}\right\|_{L^{1}} \leq\left\|u^{\delta}\right\|_{\infty} \operatorname{meas}\left(m_{\delta}\right) \leq c\|u\|_{\infty} \operatorname{meas}\left(m_{\delta}\right) .
$$

Let $x^{\delta}$ be the solution to the system

$$
\dot{x}^{\delta}(t)=f_{x}[t] x^{\delta}(t)+f_{u}[t] u^{\delta}(t), \quad x^{\delta}(0)=0 .
$$

Then, due to conditions (8.3), the pair $\xi^{\delta}=\left(x^{\delta}, u^{\delta}\right)$ belongs to $\Gamma_{0}$ and

$$
\left\|x^{\delta}\right\|_{1,1} \leq c_{f}\left\|u^{\delta}\right\|_{L^{1}} \leq c_{f} c\|u\|_{\infty} \text { meas } m_{\delta},
$$

where $c_{f}>0$ is independent from $u^{\delta}$. Since meas $\left(m_{\delta}\right) \rightarrow 0$ as $\delta \rightarrow 0+$, it follows from (8.5) and (8.6) that

$$
\left\|u^{\delta}\right\|_{L^{1}} \rightarrow 0 \quad \text { and } \quad\left\|x^{\delta}\right\|_{1,1} \rightarrow 0 \quad \text { as } \quad \delta \rightarrow 0+.
$$

According to Lemma 8.2, there exists $\delta_{1}>0$ such that

$$
T\left(\Gamma_{\delta_{1}}\right)=T\left(\Gamma_{0}\right) .
$$

Also note that, since the image $T\left(\Gamma_{\delta_{1}}\right) \subset \mathbb{R}^{k+1}$ is a subspace, there exists $c_{T}>0$ such that for any vector $z \in T\left(\Gamma_{\delta_{1}}\right)$ we can find a preimage $\xi_{z}=\left(y_{z}, u_{z}\right) \in \Gamma_{\delta_{1}}$ of this vector satisfying the estimate $\left\|\xi_{z}\right\| \leq c_{T}|z|$.

By $(8.8)$, condition $\xi^{\delta}=\left(x^{\delta}, \overline{u^{\delta}}\right) \in \Gamma_{0}$ implies that, for any $\delta \in\left(0, \delta_{1}\right)$, there exists $\left(y^{\delta}, v^{\delta}\right) \in \Gamma_{\delta_{1}}$ satisfying

$$
T\left(y^{\delta}, v^{\delta}\right)=T\left(x^{\delta}, u^{\delta}\right), \quad\left\|\left(y^{\delta}, v^{\delta}\right)\right\| \leq c_{T}\left\|T\left(x^{\delta}, u^{\delta}\right)\right\| \leq c_{T} c_{g}\left\|x^{\delta}\right\|_{\infty} .
$$

It follows from (8.7) and (8.9) that

$$
\left\|\left(y^{\delta}, v^{\delta}\right)\right\|=\left\|y^{\delta}\right\|_{1,1}+\left\|v^{\delta}\right\|_{\infty} \rightarrow 0 \quad \text { as } \quad \delta \rightarrow 0+.
$$

Set

$$
u_{\delta}=u-u^{\delta}+v^{\delta}, \quad x_{\delta}=x-x^{\delta}+y^{\delta}, \quad \xi_{\delta}=\left(x_{\delta}, u_{\delta}\right) .
$$

Then

$$
\xi_{\delta}=\xi-\xi^{\delta}+\left(y^{\delta}, v^{\delta}\right),
$$

and hence, by the first relation in (8.9), we have

$$
T \xi_{\delta}=T \xi-T \xi^{\delta}+T\left(y^{\delta}, v^{\delta}\right)=T \xi .
$$

Moreover, since $\xi^{\delta} \in \Gamma_{0}$ and $\left(y^{\delta}, v^{\delta}\right) \in \Gamma_{\delta_{1}}$, we have

$$
\varphi_{i}^{\prime}(\bar{u}(t)) u_{\delta}(t)=\varphi_{i}^{\prime}(\bar{u}(t)) u(t) \text { a.e. in } \mathcal{M}_{i 0}, \quad i=1, \ldots, q .
$$

Since $\xi=(x, u) \in \mathcal{C}(\bar{x}, \bar{u})$, relations (8.12) and (8.13) imply that $\xi_{\delta}=\left(x_{\delta}, u_{\delta}\right) \in$ $\mathcal{C}(\bar{x}, \bar{u})$. Moreover, relations (8.2) and the condition $\left(y^{\delta}, v^{\delta}\right) \in \Gamma_{\delta_{1}}$ yield

$$
\varphi_{i}^{\prime}(\bar{u}(t)) u_{\delta}(t)=0 \quad \text { a.e. in } m_{i \delta}, \quad i=1, \ldots, q .
$$


Hence condition (7.5) also holds for $\xi_{\delta}=\left(x_{\delta}, u_{\delta}\right)$. Then, by Theorem 7.5, for any $\delta \in\left(0, \delta_{1}\right)$, there exists $\left(\lambda_{\delta}, p_{\delta}, \mu_{\delta}\right) \in M^{a}(\bar{x}, \bar{u})$ such that

$$
\Omega^{a}\left(\xi_{\delta}, \lambda_{\delta}, p_{\delta}, \mu_{\delta}\right) \geq 0
$$

Set $\tilde{\xi}^{\delta}=-\xi^{\delta}+\left(y^{\delta}, v^{\delta}\right)$. Then, according to (8.11), $\xi_{\delta}=\xi+\tilde{\xi}^{\delta}$, and, by virtue of (8.4), (8.7) and $(8.10)$, for $\tilde{\xi}^{\delta}=\left(\tilde{x}^{\delta}, \tilde{u}^{\delta}\right)$ we have

$$
\left\|\tilde{x}^{\delta}\right\|_{1,1}+\left\|\tilde{u}^{\delta}\right\|_{L^{1}} \rightarrow 0 \quad \text { as } \quad \delta \rightarrow 0+, \quad \limsup _{\delta \rightarrow 0+}\left\|\tilde{u}^{\delta}\right\|_{\infty}<+\infty .
$$

Consequently,

$$
0 \leq \Omega^{a}\left(\xi_{\delta}, \lambda_{\delta}, p_{\delta}, \mu_{\delta}\right)=\Omega^{a}\left(\xi+\tilde{\xi}^{\delta}, \lambda_{\delta}, p_{\delta}, \mu_{\delta}\right)=\Omega^{a}\left(\xi, \lambda_{\delta}, p_{\delta}, \mu_{\delta}\right)+r_{\delta},
$$

where $\left|r_{\delta}\right| \rightarrow 0$ as $\delta \rightarrow 0+$. Since $M^{a}(\bar{x}, \bar{u})$ is a compact set, there exists a subsequence $\delta_{j} \rightarrow 0+$ such that $\left(\lambda_{\delta_{j}}, p_{\delta_{j}}, \mu_{\delta_{j}}\right) \rightarrow(\lambda, p, \mu) \in M^{a}(\bar{x}, \bar{u})$ as $j \rightarrow \infty$. Passing to the limit in relations (8.14) with $\delta=\delta_{j}$, we obtain that $\Omega^{a}(\xi, \lambda, p, \mu) \geq 0$. Theorem 8.1 is completely proved.

Remark 8.1. Theorem 8.1 is a consequence of [8, Theorem S.2.1], obtained by N.P. Osmolovskii using a different abstract framework. Further developments of this theorem, for problems with mixed state-control constraints, can be found in [9]-[11].

We provided above its complete proof to illustrate that the approach developed in the present work allows not only to consider more general control sets, but also to get known results when $U$ is defined by inequalities.

\section{REFERENCES}

[1] J.-P. Aubin and H. Frankowska, Set-Valued Analysis, Birkhäuser, Berlin, 1990.

[2] A. Ya. Dubovitski and A. A. Milyutin, Problems for extremum under constraints, Zh. Vychislit. Mat. i Mat. Fiz., 5, (1965), pp. 395-453; English transl. in U.S.S.R. Comput. Math. and Math. Phys. 5, (1965).

[3] H. Frankowska The maximum principle for an optimal solution to a differential inclusion with end point constraints, SIAM J. on Control and Optimization, 25, (1987), pp. 145-157.

[4] H. Frankowska, High order inverse mapping theorems, Ann. Inst. Henri Poincaré, Analyse Non Linéaire, S6, (1989), pp. 283-303.

[5] H. Frankowska, D. Hoehener and D. Tonon A second-order maximum principle in optimal control under state constraints, Serdica Mathematical Journal, 39, (2013), pp. 233-270.

[6] H. Frankowska and N. OsmolovskiI, Second-order necessary optimality conditions for the Mayer problem subject to a general control constraint, in ANALYSIS AND GEOMETRY IN Control theory and its Applications, Springer INdAM Ser., 11, Springer, Cham, 2015, pp. 171-207.

[7] D. Hoenener, Variational approach to second-order optimality conditions for control problems with pure state constraints, SIAM J. Control Optim., 50 (2012), pp. 1139-1173.

[8] E. S. Levitin, A. A. Milyutin, N. P. Osmolovskis, Conditions of high order for a local minimum in problems with constraints, Russian Math. Surveys 33, (1978), pp. 97-168.

[9] N. P. OsmolovsKiI, Necessary quadratic conditions of extremum for discontinuous controls in optimal control problem with mixed constraints, Journal of Math. Sciences, 183 (2012), pp. 435-576.

[10] N. P. OsmolovskiI, Necessary second-order conditions for a weak local minimum in a problem with endpoint and control constraints, J. Math. Anal. and Appl., 457, (2018), pp. 16131633.

[11] N. P. Osmolovskit and H. Maurer, Applications to Regular and Bang-Bang Control. Second-Order Necessary and Sufficient Optimality Conditions in Calculus of Variations and Optimal Control, SiAM, Philadelphia, PA, 2012.

[12] L. S. Pontryagin, V. G. Boltyanski, R. V. Gramkrelidze, and E. F. Miscenko, The Mathematical Theory of Optimal Processes, Pergamon Press, New York, 1964. 